Canadian Food Studies

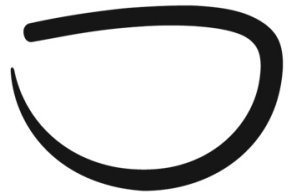

La Revue canadienne des études sur l'alimentation

Original Research Article

\title{
Supporting Inuit food security: A synthesis of initiatives in the Inuvialuit Settlement Region, Northwest Territories
}

\author{
Tiff-Annie Kennya, Sonia D. Wesche ${ }^{b^{*}}$, Myriam Fillion ${ }^{c, d}$, Jullian MacLeane, \\ Hing Man Chana \\ a Department of Biology, University of Ottawa \\ ${ }^{\mathrm{b}}$ Department of Geography, Environment and Geomatics, University of Ottawa \\ 'Science and Technology Department, TÉLUQ University, Université du Québec \\ ${ }^{d}$ Centre de recherche interdisciplinaire sur le bien-être, la santé, la société, et \\ I'environnement (CINBIOSE), Université du Québec à Montréal \\ e Inuvialuit Regional Corporation, Inuvik, NT
}

\begin{abstract}
Food insecurity among Indigenous Peoples of northern Canada is a significant public health issue that is exacerbated by changing social and environmental conditions. While a patchwork of programs, strategies and polices exist, the extent to which they address all "pillars" of food security (food availability, access, quality, and utilization) remains under-assessed. We respond to this gap by providing a framework for synthesizing and assessing information about food security initiatives, using a case study of the Inuvialuit Settlement Region (ISR), the westernmost Inuit region of Canada. Our objectives are: (1) to identify existing initiatives in the ISR; (2) to assess the breadth and diversity of these initiatives in addressing the four key food security "pillars"; and (3) to present an analytical framework that will facilitate ongoing data updating and sharing in the ISR and elsewhere. Through a scoping review and direct consultation with 12 key informants, we identified 30 initiatives that support food security in the ISR. These are funded and implemented at a range of national, territorial, regional, and local levels, and include both governmental and non-governmental programs, strategic frameworks, and research and monitoring initiatives. Seven key themes emerged from the cross-scale analysis of these initiatives, including: orientation with respect to food security pillars, scope and scale, demographic targeting, funding, monitoring and evaluation, and implications for food security
\end{abstract}


strategies. While our framework provides a useful tool for data synthesis and analysis, its outputs can help in identifying gaps and opportunities for both resource allocation and program and policy development for under-served communities. Significantly, this study highlights the importance of engaging local perspectives in the development of coordinated approaches to address Inuit food insecurity.

Keywords: Inuit; Indigenous; Canada; food insecurity; food programs; food security initiatives; program assessment

\section{Introduction}

In northern Canada, the high price of nutritious market foods, together with changing lifestyles, acculturative stresses, and access barriers to locally-harvested, culturally-preferred, country (wild) foods present a significant challenge to the food security of Indigenous Peoples (Council of Canadian Academies, 2014), with Inuit experiencing the greatest disparity relative to the general Canadian population. Many Inuit have insufficient or unpredictable access to safe, affordable, and nutritious food to meet their dietary and food preference needs; thus, they experience food insecurity. Unemployment, low incomes and high food costs are principle causes (Egeland, 2010). The 2007-2008 Inuit Health Survey (Saudny, Egeland, \& Leggee, 2012) classified 62.6 percent of Inuit households as food insecure, with Nunavut (one of the four Inuit regions in Canada) showing the highest documented prevalence (68.8 percent) among all Indigenous Peoples in a developed country (Egeland, 2011; Egeland, et al. 2011; Rosol et al., 2011). In the Inuvialuit Settlement Region (ISR), the westernmost Inuit region of Canada, 43 percent of Inuit households were classified as food insecure (Rosol et al., 2011), compared to 7.7 percent of total Canadian households (Health Canada, 2012).

Food insecurity remains an important public health issue, even in developed and foodrich countries such as Canada and the United States (Olson, 1999; Seligman, Laraia, \& Kushel, 2010; Stuff et al., 2004; Willows, Veugelers, Raine, \& Kuhle, 2011). At the sub-national level, food insecurity is disproportionately experienced by certain groups, particularly Indigenous Peoples (Egeland, Johnson-Down, Cao, Sheikh, \& Weiler, 2011; Skinner, Hanning, \& Tsuji, 2014; Rosol et al., 2011; Willows et al., Kuhle, 2011). For Inuit, adverse health effects include (but are not limited to) disrupted eating patterns, reduced diet quality and increased susceptibility to chronic and infectious disease (Egeland et al., 2011; Huet, Rosol, \& Egeland, 2012). Food security is also a social determinant of health, reflecting the underlying socioeconomic conditions that influence Inuit health outcomes (Inuit Tapiriit Kanatami, 2014).

Inuit, scholars and other national and international actors have called for immediate action to mitigate the negative health impacts of food insecurity in Canada's North (Council of Canadian Academies, 2014; De Schutter, 2012; Rosol et al., 2011). Despite diverse efforts to address the issue and its underlying causes, Inuit food insecurity rates remain high (Huet et al., 2012; Rosol et al., 2011). This highlights the need to better understand the existing program and 
policy landscape in Inuit regions_-including the scope, breadth, complementarity and cultural appropriateness of food security interventions.

\section{Determinants of food (in) security among Inuit}

The Inuit food system is comprised of three interrelated dimensions: the country (traditional, wild) food system, the market (store-bought) food system and, to a lesser extent, the locallyproduced (locally-grown) food system. Distinction among these sub-systems is complicated by factors such as the commodification of country foods (Searles, 2016). Moreover, there is complex interplay between subsistence and wage-based activities in Inuit communities, with most households participating in both and balancing the resources derived from each (Council of Canadian Academies, 2014; Condon, Collings, \& Wenzel, 1995; Natcher, 2009; Parker, 2016; Todd, 2010; Usher, 1976). Employment in the wage economy, for instance, can support country food access by providing cash for harvesting equipment and supplies while at the same time limiting the time individuals can devote to harvesting activities.

Food security is a multidimensional concept, premised on the "pillars" of sustained food availability (supply of food), food access (affordability and allocation), food quality (nutritional quality and food safety), and food utilization (food knowledge/skills and cultural preferences) (FAO, 1996; Nunavut Food Security Coalition, 2014; Wesche \& Chan, 2010). Below, we summarize the major determinants of these "pillars" for each dimension of the Inuit food system. This provides a context for our analysis of existing initiatives to promote food security, using the ISR as a case study.

\section{Country food system}

The availability of country foods is influenced by environmental and ecological conditions that shape the health, abundance, distribution, and migration of wildlife populations. Inuit communities have witnessed climate-related impacts on their wild food systems, including declines in key wildlife populations, that could have significant consequences for food security and diet quality (Guyot, Dickson, Paci, Furgal, \& Chan, 2012; Rosol, Powell-Hellyer, \& Chan, 2017; Wesche \& Chan, 2010). For example, many caribou (Rangifer tarandus) populations across the circumpolar north are experiencing dramatic declines in abundance (Gunn, Russell, \& Eamer, 2011). Related conservation measures such as harvest moratoria can further constrain country food access and affect diet quality (Chan et al., 2006; Rosol et al., 2017).

Access to country food may be influenced by changes in both environmental/ecological and social systems. Changes in the physical harvesting environment (e.g. reduced ice safety, unpredictable weather conditions) may limit harvesters' ability to safely and predictably access wildlife (Chan, 2006; Ford, 2009; Lambden, Receveur, \& Kuhnlein, 2007; Meakin \& Kurvits, 2009; Nancarrow \& Chan, 2010; Wesche \& Chan, 2010). Employment status, income, time available for harvesting, and the ability to purchase and/or maintain equipment and supplies can 
also influence the level of harvesting and consequently affect country food access (Condon, Collings, \& Wenzel, 1995; Hopping et al., 2010; Huet et al., 2012; Mackey \& Orr, 1987; Mead, Gittelsohn, Kratzmann, Roache, \& Sharma, 2010b). Where country food is available for purchase (e.g. country food markets), financial means also determine access (Lardeau, Healey, \& Ford, 2011; Myers, Powell, \& Duhaime, 2004). Other sociocultural factors, such as kinship ties, reciprocal relationships, and food sharing networks can influence country food access, and play an important role in the food security status of vulnerable community members (e.g. Elders and single mothers) (Beaumier \& Ford, 2010; Lardeau, Healey, \& Ford, 2011).

Regarding food quality, food safety is an integral dimension. While country foods are nutritious and often preferred, they are also the principal exposure vector for many persistent environmental contaminants in the Arctic (Chan, 1998; Donaldson et al., 2010; Van Oostdam et al., 2005). Although the majority of the Inuit population falls below Health Canada guideline levels for heavy metals (e.g. mercury and lead) and persistent organic pollutants, the body burden of these contaminants often exceeds that observed in the general Canadian population (Chan, 1998; Chan, Kim, Khoday, Receveur, \& Kuhnlein, 1995; Kuhnlein \& Chan, 2000; Laird, Goncharov, \& Chan, 2013). For example, average blood mercury concentration among Inuit women (18-45 years) in Nunavut was approximately eight times higher than the female Canadian national average, although still below the 8 ppb population guideline (Chan, 2012).

At the same time, for country food to remain a viable part of the food system, harvest activities must be practiced and country foods must be effectively utilized. For Inuit, subsistence activities (e.g. hunting, fishing, trapping, and gathering) remain inextricably linked to wellbeing and cultural identity (Borré, 1991; Collings, Wenzel, \& Condon, 1998; Duhaime, Chabot, \& Gaudreault, 2010; Kishigami, 2004; Searles, 2002; Wenzel, 1991). However, changes in the transfer of traditional knowledge and skills to younger generations (Pearce et al., 2011), and other acculturative stresses (e.g. declining participation in traditional activities, competing demands on time, changing food preferences) can also influence utilization, cultural preference, and taste for country foods (Willows, 2005).

\section{Market food system}

Despite the very complex and costly logistics of food retailing in northern Canada, where many small communities have limited or no road access, market food is now routinely available in remote community stores through private retail corporations or community co-operatives (Enrg Research Group, 2016). Food diversity generally remains limited, however.

While a federal subsidy program exists to mitigate high food costs across the north, concerns over retailer accountability and limited market competition remain (Burnett, Skinner, \& Leblanc, 2015; Galloway, 2014; 2017; Rennie, 2014; Skinner et al., 2016). The high cost of food in northern Canada, particularly for fresh fruit and vegetables, is well documented and remains an important access barrier to nutritious foods (Duhaime \& Caron, 2012; Lambden et al., 2006). 
For instance, in 2011 the average price of market foods in Nunavik (Inuit region of Quebec) was 81 percent higher than in the provincial capital of Quebec City (Duhaime \& Caron, 2012).

In addition to availability and access constraints, the quality of perishable foods can deteriorate significantly during long-distance transport, thus decreasing consumer preference for healthful fruit and vegetables. Furthermore, lifestyle changes that include time constraints and the appeal of convenience foods, as well as limited nutritional and food preparation knowledge regarding market foods may limit the utilization of healthful market food (Duhaime et al., 2002; Ford \& Beaumier, 2011).

\section{Locally-produced food system ${ }^{1}$}

Food production in the north is constrained by biophysical conditions (e.g. cold climate, permafrost, polar nights). Accordingly, locally-produced food has historically occupied a negligible role in the Inuit food system. However, innovative food production techniques and practices (e.g. cold climate greenhouses, community gardening and animal husbandry), may "have the potential to become key elements" in northern food strategies (Avard, 2015). Enhancing northern food production (i.e. availability) and local/regional food distribution networks (i.e. access) is a recognized priority area for economic development and food security in Canada's northern regions (GNWT, 2017; Nunavut Food Security Coalition, 2014). Locallyproduced food can improve nutritional quality through freshness, but nevertheless requires a shift in consumer food knowledge, skills and choice (i.e. utilization) and significant financial investment.

\section{Moving forward on food security}

As food security is multifaceted and complex, initiatives to address its various determinants and dimensions vary widely, ranging from short-term hunger mitigation efforts to longer-term programs and policies designed to address root causes (Barrett, 2002; Council of Canadian Academies, 2014). Over the long term, broader-scale strategies are required to support sustainable, resilient food systems that are culturally-appropriate and grounded in the principles of Indigenous food sovereignty (Grey \& Patel, 2015; Morrison, 2011; Weiler et al., 2015). Currently, multiple programs, policies and strategies are in place in the North; however, the extent to which these efforts are complementary and address all aspects of food security (i.e. access, availability, quality, and utilization) remains under-assessed. This study aims to inform some of these gaps.

In this study, we focused specifically on formalized initiatives (including programs and strategies, from national to local) aimed at supporting food security in the North, using the ISR

\footnotetext{
1 "Locally-produced food" is here understood to include any foods produced either within the community or in the broader region/territory (e.g., ISR or NWT).
} 
as a case study. Our objectives are: (1) to inventory existing (and when relevant, notable defunct) programs in the ISR, (2) to assess the breadth and diversity of these programs in addressing the four key food security pillars, and (3) to present an analytical framework that will facilitate ongoing data updating and sharing in the ISR and elsewhere. Our framework is structured around the program themes outlined in the recent expert panel report, Aboriginal Food Security in Northern Canada: An Assessment of the State of Knowledge (Council of Canadian Academies, 2014). Within each theme, results are tabulated from higher to lower scales of implementation, recognizing the diversity of funding structures (national, territorial and local), and including both governmental and non-governmental funding sources. The relevant food security pillars are indicated and discussed for each initiative.

\section{Methods}

This paper is based on information gathered in the ISR, an Inuit Land Claim Settlement area located primarily in the northernmost part of the Northwest Territories (NWT). The project emerged from ongoing collaboration between academic researchers, regional organizations and community representatives. During regional food safety and security workshops in 2012 and 2014, participants prioritized the identification of existing food security initiatives to provide a basis for developing a comprehensive food security strategy for the ISR (Fillion et al., 2014).

\section{Setting}

With a total area of 1,172,749 $\mathrm{km}^{2}$ (Inuvialuit Regional Corporation, 2017) and a population of 5,700, the ISR encompasses six primarily Inuvialuit (Inuit) communities: Aklavik, Inuvik, Paulatuk, Sachs Harbour, Tuktoyaktuk, and Ulukhaktok (Figure 1). The Inuvialuit Regional Corporation (IRC), created at the signing of the Inuvialuit Final Agreement in 1984, has a governance mandate of improving the economic, social and cultural wellbeing of Inuvialuit beneficiaries. Despite devolutionary arrangements and considerable movement towards Inuvialuit self-government, much of the jurisdictional authority to deliver programs and services resides with the Governments of NWT (GNWT) and Canada.

Inuvik (population 3,170), the only ISR community with year-round road access ${ }^{2}$, serves as the administrative center for the western Canadian Arctic and provides regional public services (e.g. high school, hospital, long-term care facility). The other five communities are smaller and more remote, with populations ranging from 117 (Sachs Harbour) to 996 (Tuktoyaktuk) (NWT Bureau of Statistics, 2016). Each ISR community has a hamlet/town office,

\footnotetext{
2 At the time that this research was conducted, an all-season highway was under construction between Inuvik and Tuktoyaktuk. The opening of the highway (in November 2017) impacts the food system. For example, food can now be brought in by truck year-round and the community no longer qualifies for the Nutrition North Canada subsidy on market food items.
} 
a Community Corporation, and a Hunters and Trappers Committee, all of which administer funding and deliver programs/services for community purposes. Other public and private services/infrastructure in these communities include schools (Beaufort Delta Education Council), community and youth centers, churches, and grocery/general stores (one or two stores per community). Approximately 70 percent of Inuvik’s population self-identified as Indigenous, compared to 83-92 percent in the smaller ISR communities (NWT Bureau of Statistics, 2016). Average family income in the region ranges from CAD \$58,958 in Ulukhaktok to CAD $\$ 112,044$ in Inuvik ${ }^{3}$ (NWT Bureau of Statistics, 2016).

Food system structure and dynamics differ markedly between Inuvik and the five smaller communities. With the exception of Inuvik, the majority of individuals in the region report that half or more of their meat consumption is country food (NWT Bureau of Statistics, 2016). Stores in the smaller communities obtain market food year-round through air shipment, and seasonally by ice road (Aklavik and Tuktoyaktuk ${ }^{3}$ only, in winter) and barge (once per year, during ice melt). The average cost of the Revised Northern Food Basket (to feed a family of four with a healthful diet for one week) in the ISR was CAD \$410 in 2014-2016, over twice the cost in Ottawa (CAD \$192), the nation’s capital. At a regional scale, country foods are obtained principally from hunting and sharing networks (among family, friends and community organizations); over two-thirds of ISR households reported sharing country food with others (Egeland, 2010).

Local-scale social, cultural and economic dynamics have important implications for the risk and experience of food insecurity, particularly in the smaller communities (Collings, 2011; Collings et al., 1998; Collings, Marten, Pearce, \& Young, 2016; Parker, 2016). For example, household structure (e.g. marital status) has implications for household centrality in community resource and food sharing networks, a traditional mechanism for maintaining food security and social relations (Collings et al., 2016). Consequently, single women and single men who lack an active hunter in the household and/or who have limited access to sharing networks may experience constrained country food access (Collings et al., 2016), which is associated with disparities in food security status (Duhaime et al., 2002, Gaudreault, 2010). While these localscale sociocultural factors can influence the implementation and viability of food security initiatives, a detailed analysis of these relationships is beyond the scope of this study.

\section{Identification of food security initiatives}

In this study, we first undertook a scoping review of the academic literature (Levac, Colquhoun, \& O'Brien, 2010) to identify publications that address food security initiatives across the four Inuit regions (for broader context), with a focus on the ISR. Searches in PubMed and Web of Science databases were conducted using the following single and combined search terms: Inuit,

\footnotetext{
${ }^{3}$ Average income in Inuvik is higher than in the remote communities due to the concentration of high-salary employment and may not be representative of Inuit household income given the town's significant non-Indigenous population.
} 
Inuvialuit, food, food security, nutrition, harvest, country food, strategy, policy, and program. We then systematically searched the websites of national, regional and community governments; non-governmental organizations; and public health agencies for relevant reports, communications and references related to food security programs and strategies. Subsequently, to verify our initial list and identify additional initiatives, we conducted a series of consultations $(n=12)$ with northern program managers, organizational representatives (national, territorial, regional and local), and community research assistants between autumn 2014 and autumn 2015. These were accomplished through a combination of in-person meetings, telephone calls, and emails. Prior to publication, we verified this information with relevant contacts and updated it as necessary.

Figure 1: Location of the Inuvialuit Settlement Region and its six communities. (Map created by Sarah Simpkin; Map data from Natural Resources Canada (2016), licensed under the Open Government Licence - Canada)

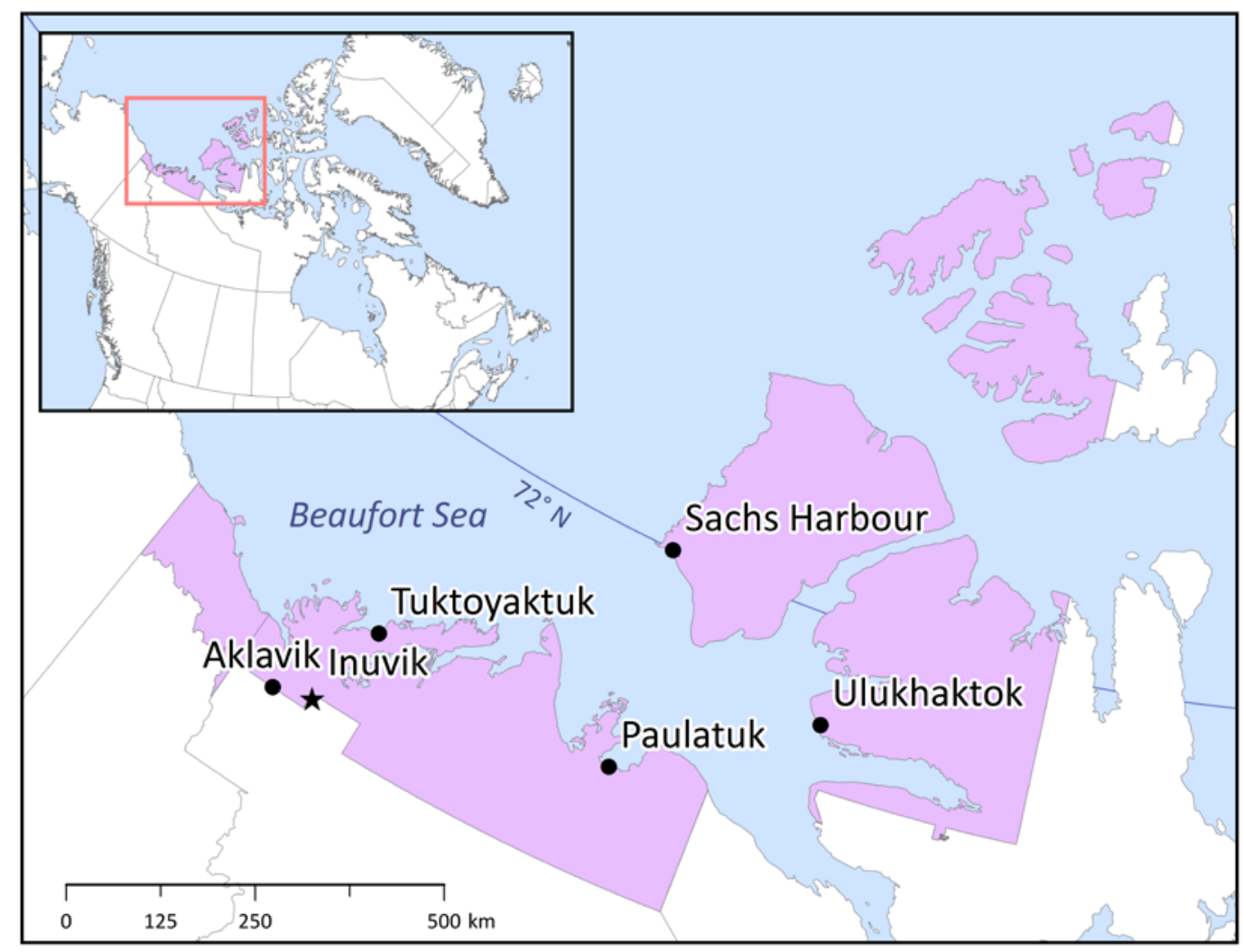

\section{Analytical framework}

To effectively synthesize and assess information about food security initiatives in the ISR, we developed an analytical framework based on: a) the seven thematic areas defined by the Council of Canadian Academies (CCA; 2014), and b) the four pillars of food security described above 
(FAO, 1996; Nunavut Food Security Coalition, 2014; Wesche \& Chan, 2010) (Table 1). First, food security initiatives were categorized from higher to lower scales of organization (i.e. national to local) based on the implementing body, under the following CCA themes: (1) affordability and availability of healthy foods; (2) health and education programs; (3) community wellness and intergenerational knowledge sharing; (4) harvester support and sustainable wildlife management; (5) poverty reduction and community economic development; (6) infrastructure, transportation and local food production; (7) youth engagement. While many initiatives intersect or overlap with multiple thematic areas outlined above, this classification scheme was the most appropriate available structure for interpreting our results. Second, each initiative was assessed to determine its contribution to addressing one or more food security pillars.

In the next section, we provide an overview of ISR food security initiatives and discuss each CCA program theme in turn. This is followed by a discussion of emergent cross-cutting themes from the analysis of these initiatives, including orientation with respect to food security pillars, scope and scale, demographic targeting, funding, monitoring and evaluation for evidencebased policy-making, and implications for food security strategies. We then conclude by highlighting the importance of understanding and assessing the evolving landscape of foodrelated initiatives to support sustainable food systems and food security over the long term.

\section{Results and Discussion}

In the ISR, we identified a total of 30 initiatives that are funded and implemented at different levels of organization (national, territorial, regional, and local) and support food security at the community or regional scale (Table 1). These include: (1) volunteer, non-profit and/or donationbased community initiatives such as food banks, (2) government and institutional programs supported by sustained core-funding allocations, (3) application-based funding opportunities (impermanent or annual), (4) strategic frameworks and action plans (e.g. anti-poverty, wellness or northern economic development), and (5) research and monitoring initiatives. We have attempted to provide a comprehensive inventory through systematic search strategies and direct consultation with northern program managers; however, the final list may not be exhaustive and will necessarily evolve over time. Particularly challenging is the comprehensive identification of impermanent community-based initiatives (such as community harvests) resultant from application-based funding (e.g. from non-profit organizations). It is important to recognize that each initiative includes a number of components (e.g. infrastructure, funding, management, community support, and strategic vision) that must work in tandem to ensure sustained program activity and support for food security. 
Table 1: Summary of Current Food Security Initiatives in the Inuvialuit Settlement Region. The initiatives are organized by Council of Canadian Academies (2014) theme, from higher to lower scales of implementation within each theme. The relevant food security pillars are indicated for each program.

\begin{tabular}{|c|c|c|c|c|c|}
\hline INITIATIVE & $\begin{array}{l}\text { IMPLEMENTING BODY } \\
\text { (FUNDING*) }\end{array}$ & LOCATION & DESCRIPTION & $\begin{array}{l}\text { TARGET } \\
\text { POPULATION }\end{array}$ & $\begin{array}{l}\text { FOOD } \\
\text { SECURITY } \\
\text { PILLAR }\end{array}$ \\
\hline \multicolumn{6}{|c|}{ Theme 1: Increasing the affordability and availability of healthful foods } \\
\hline $\begin{array}{l}\text { 1. Nutrition } \\
\text { North Canada: } \\
\text { Food Subsidy }\end{array}$ & $\begin{array}{l}\text { Registered Northern retailers, } \\
\text { Southern suppliers, and Country } \\
\text { food processors/distributors } \\
\text { (Indigenous and Northern } \\
\text { Affairs Canada) }\end{array}$ & $\begin{array}{l}\text { Five remote } \\
\text { ISR } \\
\text { communities }^{\dagger}\end{array}$ & $\begin{array}{l}\text { Subsidized transport of perishable } \\
\text { nutritious food and commercially- } \\
\text { produced country food to remote } \\
\text { northern communities }\end{array}$ & All residents & $\begin{array}{l}\text { Availability, } \\
\text { Access }\end{array}$ \\
\hline $\begin{array}{l}\text { 2. Arctic Food } \\
\text { Bank }\end{array}$ & $\begin{array}{l}\text { Midnight Sun Mosque } \\
\text { (Muslim Welfare Centre) }\end{array}$ & Inuvik & Provides food items & People in need & Access \\
\hline $\begin{array}{l}\text { 3. Inuvik Food } \\
\text { Bank }\end{array}$ & $\begin{array}{l}\text { Inuvik Food Bank } \\
\text { (Food Banks Canada) }\end{array}$ & Inuvik & Provides food items & People in need & Access \\
\hline 4. Food Bank & $\begin{array}{l}\text { Our Lady of Lourdes } \\
\text { (Society of Saint Vincent de } \\
\text { Paul) }\end{array}$ & Paulatuk & Provides food items & People in need & Access \\
\hline 5. Food Bank & $\begin{array}{l}\text { Hamlet of Sachs Harbour } \\
\text { (NWTHC: Small Community } \\
\text { Homelessness Fund) }\end{array}$ & $\begin{array}{l}\text { Sachs } \\
\text { Harbour }\end{array}$ & Provides food items & People in need & Access \\
\hline 6. Food Bank & $\begin{array}{l}\text { Our Lady of Grace Church } \\
\text { (Society of Saint Vincent de } \\
\text { Paul) }\end{array}$ & Tuktoyaktuk & Provides food items & People in need & Access \\
\hline 7. Food Bank & $\begin{array}{l}\text { Hamlet of Ulukhaktok } \\
\text { (NWTHC: Small Community } \\
\text { Homelessness Fund, Municipal } \\
\text { Funds) }\end{array}$ & Ulukhaktok & Provides food items & People in need & Access \\
\hline
\end{tabular}




\begin{tabular}{|c|c|c|c|c|c|}
\hline $\begin{array}{l}\text { 8. Soup } \\
\text { Kitchen }\end{array}$ & $\begin{array}{l}\text { Ingamo Hall Friendship Centre } \\
\text { (NWTHC: Small Community } \\
\text { Homelessness Fund) }\end{array}$ & Inuvik & Provides hot meals & People in need & Access \\
\hline $\begin{array}{l}\text { 9. Meal } \\
\text { Program }\end{array}$ & $\begin{array}{l}\text { Inuvik Homeless Shelter } \\
\text { (NWTHC: Small Community } \\
\text { Homelessness Fund) }\end{array}$ & Inuvik & Provides hot meals & $\begin{array}{l}\text { People who are } \\
\text { homeless }\end{array}$ & Access \\
\hline $\begin{array}{l}10 . \\
\text { Community } \\
\text { Kitchen }\end{array}$ & $\begin{array}{l}\text { Our Lady of Victory Roman } \\
\text { Catholic Church } \\
\text { (Society of Saint Vincent de } \\
\text { Paul, NWTHC: Small } \\
\text { Community Homelessness Fund) }\end{array}$ & Inuvik & Provides hot meals & People in need & Access \\
\hline $\begin{array}{l}11 . \\
\text { Homelessness } \\
\text { Kitchen }\end{array}$ & $\begin{array}{l}\text { Hamlet of Paulatuk } \\
\text { (NWTHC: Small Community } \\
\text { Homelessness Fund) }\end{array}$ & Paulatuk & Open kitchen and food provision & People in need & $\begin{array}{l}\text { Access, } \\
\text { Utilization }\end{array}$ \\
\hline \multicolumn{6}{|c|}{ Theme 2: Health and Education } \\
\hline $\begin{array}{l}\text { 12. Nutrition } \\
\text { North Canada: } \\
\text { Nutrition } \\
\text { Education }\end{array}$ & $\begin{array}{l}\text { Inuvialuit Regional Corporation } \\
\text { (Indigenous and Northern } \\
\text { Affairs Canada) }\end{array}$ & $\begin{array}{l}\text { Five remote } \\
\text { ISR } \\
\text { communities }^{\dagger}\end{array}$ & $\begin{array}{l}\text { Cooking circles and food demonstrations } \\
\text { carried out by a hired local community } \\
\text { member to increase knowledge of } \\
\text { healthy eating and enhance healthful } \\
\text { food preparation skills }\end{array}$ & Adults & Utilization \\
\hline $\begin{array}{l}\text { 13. Canadian } \\
\text { Pre-Natal } \\
\text { Nutrition } \\
\text { Program: First } \\
\text { Nations and } \\
\text { Inuit } \\
\text { Component } \\
\text { (Including } \\
\text { Inuvik Healthy } \\
\text { Babies) }\end{array}$ & $\begin{array}{l}\text { Inuvialuit Regional Corporation } \\
\text { (Public Health Agency of } \\
\text { Canada) }\end{array}$ & $\begin{array}{l}\text { All six ISR } \\
\text { communities }\end{array}$ & $\begin{array}{l}\text { Provides support for various programs } \\
\text { including maternal nourishment and } \\
\text { food provision (cooking, snacks, food } \\
\text { coupons/vouchers and baskets), } \\
\text { nutritional education and breastfeeding, } \\
\text { and country food preparation }\end{array}$ & $\begin{array}{l}\text { Pregnant women, } \\
\text { mothers of infants, } \\
\text { and infants up to } 12 \\
\text { months; in particular } \\
\text { those identified as } \\
\text { high risk }\end{array}$ & $\begin{array}{l}\text { Access, } \\
\text { Utilization }\end{array}$ \\
\hline
\end{tabular}




\begin{tabular}{|c|c|c|c|c|c|}
\hline $\begin{array}{l}\text { 14. Northern } \\
\text { Contaminants } \\
\text { Program }\end{array}$ & $\begin{array}{l}\text { Partnership between } \\
\text { Community organizations, } \\
\text { Researchers and Governments at } \\
\text { various levels } \\
\text { (Indigenous and Northern } \\
\text { Affairs Canada) }\end{array}$ & $\begin{array}{l}\text { All six ISR } \\
\text { communities }^{\ddagger}\end{array}$ & $\begin{array}{l}\text { Provides funding for research, } \\
\text { monitoring, and communication to } \\
\text { enhance understanding of the } \\
\text { benefit/risks of country food } \\
\text { consumption and support informed food } \\
\text { choices }\end{array}$ & All residents & Quality \\
\hline $\begin{array}{l}\text { 15. Nutrition } \\
\text { education and } \\
\text { school } \\
\text { snack/meal } \\
\text { programs }\end{array}$ & $\begin{array}{l}\text { Schools } \\
\text { (Breakfast for Learning Canada, } \\
\text { Food First Foundation, IRC: } \\
\text { Healthy Living and Disease } \\
\text { Prevention) }\end{array}$ & $\begin{array}{l}\text { All six ISR } \\
\text { communities }^{\ddagger}\end{array}$ & $\begin{array}{l}\text { Helps start and sustain school-based } \\
\text { meal and snack programs, including } \\
\text { funding for food, supplies and } \\
\text { equipment (e.g. kitchen and garden } \\
\text { equipment, cold storage), and } \\
\text { staff/volunteer support, as well nutrition } \\
\text { education programming }\end{array}$ & $\begin{array}{l}\text { School-aged children } \\
\text { and adolescents }\end{array}$ & $\begin{array}{l}\text { Availability, } \\
\text { Access, } \\
\text { Utilization }\end{array}$ \\
\hline $\begin{array}{l}\text { 16. Drop the } \\
\text { Pop NWT }\end{array}$ & $\begin{array}{l}\text { Schools } \\
\text { (GNWT: Health and Social } \\
\text { Services) }\end{array}$ & $\begin{array}{l}\text { All six ISR } \\
\text { communities }^{\ddagger}\end{array}$ & $\begin{array}{l}\text { School-based educational campaign and } \\
\text { funding initiative to support } \\
\text { consumption of healthful foods and } \\
\text { beverages, and improving nutritional } \\
\text { knowledge and skills }\end{array}$ & $\begin{array}{l}\text { Students, families, } \\
\text { schools and } \\
\text { communities }\end{array}$ & $\begin{array}{l}\text { Access, } \\
\text { Utilization }\end{array}$ \\
\hline $\begin{array}{l}\text { 17. Healthy } \\
\text { Family } \\
\text { Program } \\
\text { (Several) }\end{array}$ & $\begin{array}{l}\text { Arctic Family Centre } \\
\text { (Beaufort Delta Health and } \\
\text { Social Services) }\end{array}$ & Inuvik & $\begin{array}{l}\text { Delivers activities and provides support } \\
\text { to enhance child and family } \\
\text { development (includes the Collective } \\
\text { Kitchen, Baby Food and Family Meal } \\
\text { programs) }\end{array}$ & $\begin{array}{l}\text { Families (prenatal to } \\
\text { age } 6 \text { ) }\end{array}$ & $\begin{array}{l}\text { Access, } \\
\text { Utilization }\end{array}$ \\
\hline \multicolumn{6}{|c|}{ Theme 3: Community wellness and intergenerational knowledge sharing } \\
\hline $\begin{array}{l}18 . \\
\text { Community } \\
\text { Wellness Plans } \\
(2013)\end{array}$ & $\begin{array}{l}\text { ISR communities in partnership } \\
\text { with the Inuvialuit Regional } \\
\text { Corporation } \\
\text { (GNWT: Health and Social } \\
\text { Services) }\end{array}$ & $\begin{array}{l}\text { All six ISR } \\
\text { communities }\end{array}$ & $\begin{array}{l}\text { Outlines community perceptions } \\
\text { regarding how current health and } \\
\text { wellness programs are faring and } \\
\text { provides a roadmap for prioritizing } \\
\text { initiatives in support of community } \\
\text { health and wellness }\end{array}$ & All residents & $\begin{array}{l}\text { Access, } \\
\text { Utilization }\end{array}$ \\
\hline $\begin{array}{l}\text { 19. Project } \\
\text { Jewel }\end{array}$ & $\begin{array}{l}\text { Inuvialuit Regional Corporation } \\
\text { (Various) }\end{array}$ & $\begin{array}{l}\text { All six ISR } \\
\text { communities }\end{array}$ & On-the-land after care wellness program & $\begin{array}{l}\text { After-care } \\
\text { participants (youth } \\
\text { and adults) }\end{array}$ & $\begin{array}{l}\text { Access, } \\
\text { Utilization }\end{array}$ \\
\hline
\end{tabular}




\begin{tabular}{|c|c|c|c|c|c|}
\hline \multicolumn{5}{|c|}{ Theme 4: Harvester support and sustainable wildlife management } & \multirow[b]{2}{*}{ Access } \\
\hline $\begin{array}{l}20 . \\
\text { Community } \\
\text { Harvesters } \\
\text { Assistance } \\
\text { Program }\end{array}$ & $\begin{array}{l}\text { Community HTCs } \\
\text { (GNWT: Environment and } \\
\text { Natural Resources) }\end{array}$ & $\begin{array}{l}\text { All six ISR } \\
\text { communities }^{\ddagger}\end{array}$ & $\begin{array}{l}\text { Provides funding to HTCs to support } \\
\text { community harvests activities (e.g. } \\
\text { purchase of harvest equipment and } \\
\text { supplies) }\end{array}$ & Harvesters & \\
\hline $\begin{array}{l}\text { 21. Inuvialuit } \\
\text { Harvesters } \\
\text { Assistance } \\
\text { Program }\end{array}$ & $\begin{array}{l}\text { Community HTCs } \\
\text { (Inuvialuit Regional } \\
\text { Corporation) }\end{array}$ & $\begin{array}{l}\text { All six ISR } \\
\text { communities }^{\ddagger}\end{array}$ & $\begin{array}{l}\text { Provides ongoing funding to support } \\
\text { Inuvialuit subsistence harvesters }\end{array}$ & $\begin{array}{l}\text { Inuvialuit } \\
\text { beneficiaries } \\
\text { (preference for } \\
\text { subsistence } \\
\text { harvesters) }\end{array}$ & Access \\
\hline $\begin{array}{l}22 . \\
\text { Community } \\
\text { Freezer }\end{array}$ & $\begin{array}{l}\text { Hamlet of Paulatuk, Paulatuk } \\
\text { HTC, Paulatuk Community } \\
\text { Corporation (Various) }\end{array}$ & Paulatuk & Provides cold-storage for country food & $\begin{array}{l}\text { Harvesters and people } \\
\text { in need }\end{array}$ & Access \\
\hline $\begin{array}{l}23 . \\
\text { Tuktoyaktuk } \\
\text { Ice House }\end{array}$ & $\begin{array}{l}\text { Hamlet of Tuktoyaktuk } \\
\text { (Various) }\end{array}$ & Tuktoyaktuk & Provides cold-storage for country food & Harvesters & Access \\
\hline \multicolumn{6}{|c|}{ Theme 5: Poverty reduction and community economic development } \\
\hline $\begin{array}{l}\text { 24. Anti- } \\
\text { Poverty Fund }\end{array}$ & $\begin{array}{l}\text { Indigenous Governments in the } \\
\text { NWT, Community Governing } \\
\text { Authorities, NGOs partnered } \\
\text { with an Indigenous or } \\
\text { Community Governing } \\
\text { Authority } \\
\text { (GNWT: Health and Social } \\
\text { Services) }\end{array}$ & $\begin{array}{l}\text { All six ISR } \\
\text { communities }^{\ddagger}\end{array}$ & $\begin{array}{l}\text { Provides application-based funding for } \\
\text { projects to combat poverty in five of the } \\
\text { Territorial Anti-Poverty Strategy Pillars } \\
\text { (child and family support; healthy living } \\
\text { and reaching potential; safe and } \\
\text { affordable housing; sustainable } \\
\text { communities; integrated continuum of } \\
\text { service) }\end{array}$ & All residents & Access \\
\hline $\begin{array}{l}\text { 25. Territorial } \\
\text { Housing } \\
\text { Programs } \\
\text { (Several) }\end{array}$ & $\begin{array}{l}\text { NWTHC } \\
\text { (NWTHC) }\end{array}$ & $\begin{array}{l}\text { All six ISR } \\
\text { communities }\end{array}$ & $\begin{array}{l}\text { Supports home ownership ( } 2 \text { programs), } \\
\text { repair and maintenance ( } 5 \text { programs), } \\
\text { and public housing }\end{array}$ & $\begin{array}{l}\text { Eligible individuals } \\
\text { based on NWT } \\
\text { Residential Tenancies } \\
\text { Act }\end{array}$ & Access \\
\hline
\end{tabular}




\begin{tabular}{|c|c|c|c|c|c|}
\hline $\begin{array}{l}\text { 26. Country } \\
\text { Food } \\
\text { Development } \\
\text { and Value- } \\
\text { added } \\
\text { Processing } \\
\text { Initiative: } \\
\text { Country Food } \\
\text { Processing } \\
\text { Methods } \\
\text { Training } \\
\text { Course }\end{array}$ & $\begin{array}{l}\text { Aurora College, Inuvialuit } \\
\text { Regional Corporation: ICEDO } \\
\text { (IRC, Gwitch'in Tribal Council, } \\
\text { GNWT: Education, Culture and } \\
\text { Employment, GNWT: Industry, } \\
\text { Tourism and Investment) }\end{array}$ & $\begin{array}{l}\text { All six ISR } \\
\text { communities }\end{array}$ & $\begin{array}{l}\text { This course teaches the knowledge and } \\
\text { skills required for value-added } \\
\text { processing of country food through in- } \\
\text { class and hands-on instruction }\end{array}$ & Adults & Utilization \\
\hline \multicolumn{6}{|c|}{ Theme 6: Innovation in infrastructure, transportation and local food production } \\
\hline $\begin{array}{l}\text { 27. Territorial } \\
\text { Agri-Food } \\
\text { Programs } \\
\text { (Several) }\end{array}$ & $\begin{array}{l}\text { GNWT: Industry, Tourism and } \\
\text { Investment } \\
\text { (Canadian Agricultural } \\
\text { Partnership) }\end{array}$ & $\begin{array}{l}\text { All six ISR } \\
\text { communities }^{\ddagger}\end{array}$ & $\begin{array}{l}\text { Provides a suite of programs and funding } \\
\text { to support training, skills-development } \\
\text { (e.g. Agriculture Training Program, } \\
\text { Agriculture and Food Processing } \\
\text { Development Program), research } \\
\text { (Agriculture and Agri-Food Research } \\
\text { Program), marketing (Market } \\
\text { Development Program) and food safety } \\
\text { (Food Safety Program) for the } \\
\text { establishment and development of the } \\
\text { NWT agriculture and agri-foods sector }\end{array}$ & NWT agri-business & $\begin{array}{l}\text { Availability, } \\
\text { Quality }\end{array}$ \\
\hline $\begin{array}{l}\text { 28. Beaufort } \\
\text { Delta Small } \\
\text { Scale Foods } \\
\text { Program }\end{array}$ & $\begin{array}{l}\text { Community Garden Societies } \\
\text { with support from the Inuvik } \\
\text { Community Greenhouse } \\
\text { (GNWT: Industry, Tourism and } \\
\text { Investment) }\end{array}$ & $\begin{array}{l}\text { Five remote } \\
\text { ISR } \\
\text { communities }^{\dagger}\end{array}$ & $\begin{array}{l}\text { Provides funding and support for the } \\
\text { installation and establishment of gardens } \\
\text { and greenhouses, as well as information } \\
\text { and skills seminars (e.g. food } \\
\text { preservation) }\end{array}$ & $\begin{array}{l}\text { All interested } \\
\text { residents }\end{array}$ & $\begin{array}{l}\text { Availability, } \\
\text { Access }\end{array}$ \\
\hline $\begin{array}{l}\text { 29. Inuvik } \\
\text { Community } \\
\text { Greenhouse }\end{array}$ & $\begin{array}{l}\text { Community Garden Society of } \\
\text { Inuvik } \\
\text { (Contribution Agreements, } \\
\text { Memberships, Fundraising) }\end{array}$ & Inuvik & $\begin{array}{l}\text { Makes greenhouse garden plots ( } 74 \text { full- } \\
\text { size plots) available to residents of } \\
\text { Inuvik }\end{array}$ & $\begin{array}{l}\text { All interested } \\
\text { residents }\end{array}$ & $\begin{array}{l}\text { Availability, } \\
\text { Access }\end{array}$ \\
\hline
\end{tabular}




\begin{tabular}{|l|l|l|l|l|l|}
\hline \multicolumn{3}{|l|}{ Theme 7: Youth engagement } & \multicolumn{2}{l|}{} \\
\hline $\begin{array}{l}\text { 30. Traditional } \\
\text { Harvest }\end{array}$ & $\begin{array}{l}\text { Schools and Indigenous } \\
\text { Program: Take } \\
\text { a Kid }\end{array}$ & $\begin{array}{l}\text { All six ISR } \\
\text { communities }\end{array}$ & $\begin{array}{l}\text { Provides funding to organize youth on- } \\
\text { (GNW-land skills training }\end{array}$ & School-aged children & $\begin{array}{l}\text { Access, } \\
\text { Utilization }\end{array}$ \\
Harvesting & Natural Resources) & & & \\
$*$
\end{tabular}

Program funding is challenging to track (particularly for initiatives that lack core, multi-year funding) given the multiplicity of funding sources and fluctuations in annual availability. Access to funding may also be influenced by local human capacity for identifying opportunities and developing funding applications.

Furthermore, program implementation may also rely on donations, fundraising, and volunteers. As such, funding sources summarized here are not necessarily comprehensive.

${ }^{\dagger}$ The five remote communities that lack year-round surface transportation include: Aklavik, Paulatuk, Sachs Harbour, Tuktoyaktuk and Ulukhaktok.

${ }^{\ddagger}$ Based on program eligibility (implementation of program may vary between communities)

Acronyms: GNWT = Government of the Northwest Territories; HTC = Hunters and Trappers Committee; ICEDO = Inuvialuit Community Economic

Development Organization (part of the Inuvialuit Regional Corporation); ISR = Inuvialuit Settlement Region; NGO= Non-governmental organizations; NWT =

Northwest Territories; NWTHC =Northwest Territories Housing Corporation 


\section{Theme 7: Increasing the affordability and availability of healthful foods}

Initiatives aimed at increasing the affordability and availability of healthful foods focus on lowering food cost and addressing the barriers that limit healthful food availability in northern communities. Programs in this category are generally geared toward market foods, although country foods may be included in some programming based on availability.

\section{Food subsidy programs}

Since the 1960s, the Government of Canada has reinforced access to nutritious market foods in remote northern communities by subsidizing food shipping costs. Notably, the now-defunct federal Food Mail Program (FMP) provided a subsidized rate on northern food shipments between 1999 and 2011. Its successor, Nutrition North Canada (NNC), was launched in 2011 as a market-driven program, providing subsidies to retailers operating in over 100 isolated northern communities across the country. Available in the five remote ISR communities, the NNC subsidy ranges from CAD $\$ 1.60$ to $\$ 6.10 / \mathrm{kg}$ for level 1 foods (nutritious perishable items) and from CAD $\$ 0.05 / \mathrm{kg}$ to $\$ 4.30 / \mathrm{kg}$ for level 2 foods (non-perishable staple items), depending on community characteristics (Government of Canada, 2017).

\section{Community food support programs}

Formal community food support programs (i.e. food-based hunger mitigation programs) are relatively new in the north, where strong cultures of reciprocity and food sharing have traditionally supported food access for those in need (Natcher, 2009; Wenzel, 1995). While community food programs are now widely used in major Arctic population centers, their role in smaller community contexts has not been comprehensively assessed (Ford, Lardeau, \& Vanderbilt, 2012; Ford, Lardeau, Blackett, Chatwood, \& Kurszewski, 2013; Lardeau et al., 2011).

A number of local-scale food support programs are offered in the ISR, including food banks, soup kitchens and other hot meal providers. Food banks are operational in five of the six ISR communities. In Inuvik, several meal programs are regularly available for people who are homeless or otherwise in need. In the ISR, food support programs are generally implemented locally by hamlet offices, not-for profit groups or charitable organizations and are often funded through donations and fundraising; thus, operations (e.g. program schedule, outreach, participant eligibility) are highly variable between programs and communities. In small communities where only one such program may exist, inconsistent funding and operational capacity may continue to leave gaps in emergency food access provision. Moreover, while such programs increase food access, they do not address the root causes of food insecurity (Riches, 2003); without adequate policies and complementary initiatives, program users may become chronically reliant, as is the case in Inuvik (Ford, Lardeau et al., 2013). 


\section{Theme 2: Health and Education}

Healthy dietary choices rely in part on nutritional knowledge and food preparation skills (Council of Canadian Academies, 2014). As such, education and capacity-building programs are an effective mechanism for improving knowledge about food, health and wellness. These initiatives may focus specifically on conveying nutrition-related information, or on capacity development activities related to food preparation, food safety and budgeting, among others.

\section{Nutrition education and food preparation programs}

Currently, NNC includes a nutrition education component that builds community knowledge of healthy eating and food preparation skills. Available in the five remote ISR communities, the program includes cooking circles and food demonstrations carried out by a local community member. Nutrition education programs (such as the federally-funded Canada Prenatal Nutrition Program (CPNP) and the Healthy Family Program (Beaufort Delta Health and Social Services)) often target families with infants/young children to encourage breastfeeding and healthful food preparation, including country food preparation. These programs may also facilitate food access (theme 1) by providing direct food support through the provision of hampers and meal ingredients.

Additional nutrition education programs targeted at children/youth and administered in school settings are funded by charitable organizations (e.g. Food First Foundation; Breakfast for Learning) and the territorial government (e.g. Drop the Pop Campaign). Each ISR community has active school-based meal and snack programs that both improve access to healthful food (theme 1) and help build nutritional knowledge and skills (themes 2 and 7). School-based food programs have had demonstrable benefits on food and nutrient intake among Indigenous youth in remote northern Ontario (Gates, Hanning, Gates, Stephen, \& Tsuji, 2016; M. Gates, Hanning, Gates, McCarthy \& Tsuji, 2013; Skinner, Hanning, Metatawabin, Martin, \& Tsuji, 2012); however, their impact among Inuit youth has not been reported.

One of the best-documented research-related health intervention programs among Inuit is Healthy Foods North (HFN) ${ }^{4}$, a multi-institutional chronic disease prevention program implemented in 2008-2009 in selected communities of the ISR and Nunavut (Sharma, 2010; Sharma, Gittelsohn, Rosol, \& Beck, 2010). The intervention aimed to promote physical activity and improve diets by supporting the consumption of fruit, vegetables and country food, while also decreasing the consumption of processed foods high in sugar and/or fat (Sharma, 2010; Sharma et al., 2010). The Inuvialuit component of HFN included three of the six ISR communities, where two received the intervention and one served as a control group with delayed intervention. Program impacts were evaluated in relation to psychosocial (Mead, Gittelsohn, De Roose, \& Sharma, 2010a; Mead, Gittelsohn, Roache, Corriveau, \& Sharma, 2013)

\footnotetext{
${ }^{4}$ HFN is no longer active and is thus not included in Table 1.
} 
socio-economic (Erber et al., 2010), healthy eating behaviour, and diet quality outcomes (Bains et al., 2014; Kolahdooz, Butler, et al., 2014; Zotor et al., 2012). Overall, HFN showed some success in mitigating the negative impacts of the nutrition transition among Inuvialuit. It also highlighted the need to tailor public health interventions and policy to local needs by using population-specific tools (Kolahdooz, Pakseresht, et al., 2014).

\section{Food safety programs}

Various programs support food security (food quality dimension) by enhancing community knowledge and capacity surrounding food safety, including the presence of environmental contaminants and zoonotic diseases in country foods. The Northern Contaminants Program (NCP) was established in 1991 to research and monitor long-range contaminants in country food species in Northern Canada. Its four subprograms (Health; Environmental Monitoring and Research; Community-Based Monitoring and Research; and Communications, Capacity and Outreach) collectively provide data (e.g. temporal trends of contaminant levels in specific country food species, human biomonitoring) to improve understanding of the health effects and benefits/risks of country food consumption and help support informed food choices. The NCP community-based monitoring program also enhances community research capacity and youth engagement (theme 7) by directly involving youth.

A number of related initiatives also offer capacity building opportunities (including knowledge transfer and hands-on skills development) for safe food handling, including for country food (see Country Food Development and Value-added Processing Initiative below).

\section{Theme 3: Community wellness and intergenerational knowledge Sharing}

Socioeconomic conditions are central to food security in the ISR, particularly in the smaller hamlets (Collings, 2011; Parker, 2016; Todd, 2010). Accordingly, food security can be supported through initiatives that bolster community wellness and intergenerational well-being. Such programs may encourage food sharing, promote the transmission of inter-generational knowledge and skills, or include community-driven food assessment or asset mapping activities (McTavish, Furgal, Popp, \& McCarney, 2012). Initiatives such as Take a Kid

Trapping/Harvesting provide a context where youth and Elders interact around country food. Such programs may also target specific groups that may be at higher risk of food insecurity. Project Jewel (IRC), for instance, is a wellness program that incorporates clinical support with on-the-land camps and culture-based activities - including food procurement and sharing - to enhance and connect people with Elders and their culture.

Various IRC initiatives assist ISR communities in supporting collective health and wellbeing. In 2013 each community developed a wellness plan based on community consultations lead by IRC in partnership with Health Canada (Inuvialuit Regional Corporation, 2013a; 2013b; 2013c; 2013d; 2013e; 2013f). Community wellness plans and activities vary among communities, 
but generally include support for culture, traditional activities, and other health-promoting activities including (but not limited to): diabetes workshops, fitness activities, community kitchens, community gardens, and school food programs and policies. As such, while food security is not a specific area of focus, the wellness plans provide a roadmap for communitybased activities and locally-identified priorities in support of community health.

\section{Theme 4: Harvester support and sustainable wildlife management}

The country food system requires that healthy wildlife populations be sustained over time, and that harvesters are able to access them. As such, it is important that harvester support programs and wildlife management programs work to balance sustainable harvesting and conservation principles. Community-based monitoring is a useful mechanism for periodically evaluating the health and population status of key species, which influences harvesting recommendations (Council of Canadian Academies, 2014).

\section{Harvester support}

Traditional harvesting practices and sustainable country food harvest can be supported through the provision of funding and materials (e.g. harvesting equipment and supplies) to individual harvesters or to community organizations (e.g. for community hunts or community freezers). As a component of land claim agreements, Canada's territorial governments deliver harvester support programs to encourage traditional harvesting activities and the production and consumption of country food. Additionally, the IRC administers the Inuvialuit Harvesters Assistance Program, which provides assistance to subsistence harvesters. Harvester support programs range from providing funding for harvesting supplies and equipment, search and rescue services, harvester salaries, community harvests, the purchase of country food for community purposes, and youth engagement and skills development activities (theme 7). Harvest-support programs are locally administered through regional or community-based hunters and trappers committees. Accordingly, decisions regarding program eligibility, funding allocation and the nature of programming are variable between communities and over time. Funding for such programs, although sustained, is limited and therefore provided on an intermittent basis and/or to a limited number of harvesters (Ford, Smit, \& Wandel, 2006; Gombay, 2009).

\section{Sustainable wildlife management}

Wildlife management policies have significant impacts on country food availability and accessibility, and consequently on food security and sovereignty (Chan et al., 2006; Ford, McDowell et al., 2013). Comprehensively addressing wildlife management policies/programs is beyond the scope of this paper; however, it is important to recognize the role of regional and 
community-based harvester committees and organizations and territorial and federal wildlife management regimes in supporting country food security. The GNWT: ENR provides funding to support community-based organizations representing the interests of hunters and trappers (Local Wildlife Committees). In turn, Inuvialuit harvester organizations provide critical knowledge and insights on matters relating to wildlife management and conservation in the region. Nonetheless, population status and harvest level information may be insufficient for rigorous decision-making in the North (Giroux, Campbell, Dumond, \& Jenkins, 2012), pointing to an ongoing need to link decision-makers across scales (national, territorial, regional, and local) and sectors (economic development and poverty reduction, public health, education and wildlife management) to support sustainable country food harvests and access (Theriault, 2011). To date however, only limited research has looked at how wildlife management and country food harvest programs and policies in the Canadian Arctic can mutually support food security and ecological sustainability (Kenny \& Chan, 2017).

\section{Community country food storage}

Community food storage programs are typically established to provide a country food "access point” for residents who are unable to harvest or have limited sharing networks (Organ, 2012). Commonly, local harvesters stock the food storage units (Boult, 2004), which include belowground “ice houses" for cold storage (passive cooling) and electrical freezers. While some programs provide financial support or purchase meat directly from harvesters, others rely on voluntary donations. Community freezers are perceived to support country food access, including extending availability both seasonally and in the face of environmental change (Chan et al., 2006; Duhaime, Chabot, \& Gaudreault, 2002; Furgal \& Seguin, 2006); however, their direct influence on food access, food security and country food consumption has received limited attention to date (Organ, 2012; Organ, Castleden, Furgal, Sheldon, \& Hart, 2014). It is important to note that program outcomes may differ between communities and across regions due to variation in organizational structure and operations. As such, literature pertaining to freezer programs from other Inuit regions (Organ, 2012; Organ, Castleden, Furgal, Sheldon, \& Hart, 2014) may not be applicable in the ISR context.

In the ISR, we identified two types of infrastructure with semi-active programs: an ice house in Tuktoyaktuk (constructed in the 1960s) and a community freezer in Paulatuk (from a GNWT-sponsored freezer program in the 1980s). The sustainability challenges experienced by these and similar defunct programs in other communities highlight the need for detailed evaluations to better understand program dynamics. This is particularly true in the face of recent renewed interest and government funding initiatives to both support new community freezer programs and regenerate existing and defunct ones (Organ, 2012). At the same time, household chest freezers provide an alternative to the communal storage model, and ISR residents have had periodic access to programs that support individual freezer acquisition. As previously stated, to be viable and provide effective support for food security, multiple dimensions of a program must 
successfully operate in tandem over time (e.g. infrastructure, funding, management, local support, and strategic vision).

\section{Theme 5: Poverty reduction and community economic development}

The mixed economic system in northern Canada has both positive and negative impacts on food security. Northerners are burdened with comparatively higher rates of unemployment and reliance on public housing, as well as lower health and education status. Characteristics of socioeconomic disadvantage (e.g. non-completion of secondary education, low income, household crowding, single parent households, household members on income support, the need to support other family members, public housing, and housing in need of major repairs) have been associated with food insecurity among Inuit (Egeland, Williamson-Bathory, Johnson-Down, \& Sobol, 2011; Ford \& Beaumier, 2011; Huet, Rosol, \& Egeland, 2012). Poverty reduction activities that relate directly to food security tend to be twofold. These include direct interventions related to income and housing, and longer-term initiatives to promote self-reliance through community economic development.

\section{Poverty reduction}

At the territorial level, the GNWT established an Anti-Poverty Strategy and Action Plan for 2014-2016 (GNWT, 2013, 2014). The associated Anti-Poverty Fund provides CAD \$1,000,000 annually to organizations and community governments for relevant projects. Food security is included under two of the Action Plan pillars: Children and Family Support, and Sustainable Communities (GNWT, 2014). Several ISR projects have received support from the fund, including community harvests that engage youth, homeless persons, and other individuals in need. Funding for such programs, however, is application-based and annual, which may limit the pool of potential applicants and the reliability of support over time.

Various types of income and social support programs exist in the ISR to mitigate the high cost of living, notably: northern tax benefits, employment insurance, housing support, and childcare programs. These programs may interact indirectly with individual and/or household level food insecurity by liberating stressed financial resources (Council of Canadian Academies, 2014). For example, affordable housing, identified as a key issue during the recent ISR food security planning process (Fillion et al., 2014), is addressed by a complement of GNWT programs.

\section{Community economic development}

The Inuvialuit Community Economic Development Organization (ICEDO), a subsidiary of the IRC, delivers numerous projects and programs to support ISR communities in fostering sustainable economic development and access to economic opportunities for Inuvialuit 
beneficiaries. ICEDO recently initiated the Country Food Development and Value-added Processing Initiative to improve regional capacity for increasing the shelf-life of country foods with the potential for making these products market-ready. This project includes a purpose-built, mobile country food processing training facility and a hands-on methods course offered in conjunction with Aurora College. The course teaches knowledge and skills related to maximizing the commercial viability of country foods.

On the retail side, food co-operatives are social enterprises that foster local control over food retailing, and offer an outlet for local, commercially harvested and value-added country foods (Islam \& Berkes, 2016). The GNWT provides support to aspiring and existing Cooperative Associations in the ISR (and across the NWT).

\section{Theme 6: Innovation in infrastructure, transportation and local food production}

In the North, multiple initiatives are designed to facilitate the logistics of food production, transportation, storage and sale of both locally/regionally-produced food and country food.

\section{Infrastructure and transportation}

In the five remote ISR communities, the majority of market food items are flown in by commercial airlines. Seasonal infrastructure and transportation services such as ice-roads (between Inuvik and Aklavik/Tuktoyaktuk) and marine transport (barge) periodically provide a lower cost alternative to air freight; however, these depend on water levels; climatic, weather, and sea-ice conditions; and continued service provision. Innovative solutions to attenuate high shipping, operating and other logistical costs are needed. Likewise, strategies to enhance local food production and distribution must address similar barriers.

\section{Local food production}

Interest in local food production is increasing in many parts of the north, including the NWT (Johnston \& Williams, 2017). Following a series of public engagement meetings, the GNWT launched the first-ever territorial Agriculture Strategy in 2017 - The Business of Food: A Food Production Plan (GNWT, 2017), which includes actions under six pillars (Planning; Community Leadership, Partners and Collaboration; Regulatory Measures; Training and Capacity Building; Resources; and Food Production). Complementarily, the Canadian Agricultural Partnership funds several programs to support the establishment and development of the NWT agriculture and agri-foods sector (i.e. local food production, distribution, and sales). These include skillsdevelopment (e.g. Agriculture Training Program, Agriculture and Food Processing Development Program), research (Agriculture and Agri-Food Research Program), marketing (Market Development Program), food safety (Food Safety), and garden and greenhouse establishment (Small Scale Foods Program) programs. In the remote ISR communities, small greenhouses were 
established in 2016 and local garden societies were tasked with program development in consultation with the Inuvik Greenhouse Coordinator. In Inuvik, a community greenhouse has been running since 1998, supported by membership fees, fundraising and additional intermittent sources.

\section{Country food exchange}

The commodification of country foods (including commercial harvests/fisheries, country food stores/markets) is posed as a strategy to support broader access, particularly in larger settlements and for households that lack hunters or food sharing networks (Duhaime et al., 2002; Ford, Macdonald, Huet, Statham, \& MacRury, 2016). Historically, country food commodification programs largely resulted in exports to non-Inuit markets and, despite providing economic benefits to communities (Duhaime et al., 2002; Whittles, 2014), appear to have had negligible impacts on local country food access and food security (Council of Canadian Academies, 2014). In the ISR, muskox (Ovibos moschatus) has been commercially harvested on Banks Island (near Sachs Harbour) since 1981 for export to domestic and international markets (Whittles, 2014). Additionally, meat from Canadian Reindeer ${ }^{5}$, a privately-owned company in the Inuvik area, is distributed annually to Inuvialuit beneficiaries and is also available on the commercial market.

As the potential for enhanced country food commodification is currently being explored in the ISR, the development of capacity, knowledge and skills to support such efforts is progressing via the mobile country food processing training facility and methods course described above. While it is recognized that commodification could play a role in enhancing country food access and availability in the region and across the North, the diversity of local perspectives on this issue, including concerns regarding impacts on community sharing networks, which support food security and remain fundamental to Inuit social relations (Collings et al., 2016); the potential exclusion of vulnerable community members through prohibitive pricing (Myers, 2002; Lardeau, Healey, \& Ford, 2011); and regulatory and sustainability issues related to wildlife harvest present unresolved challenges.

More informally, country food is also bartered and bought/sold within Inuit communities and regions through local or regional organizations and businesses, and through social networks, including via Facebook. In the ISR, airlines offer a subsidized rate for country food transport that may facilitate these types of exchanges.

\footnotetext{
${ }^{5}$ A herd of reindeer was introduced to the region in the 1930s to mitigate against caribou shortages. While reindeer (Rangifer tarandus) are of the same genus as the culturally-valued caribou, they are semi-domesticated and actively managed with husbandry practices.
} 


\section{Theme 7: Youth engagement}

Recognizing that youth are the group most impacted by the nutrition transition and also future community leaders, their engagement is essential for building food security in the North. Youth engagement is often linked with intergenerational knowledge transmission, food-related skills development, and other educational aspects. While this theme overlaps with several aforementioned themes, it is included as a stand-alone category to highlight the key role of this particular group in building sustainable and sovereign food systems.

The Take a Kid Trapping/Harvesting Program provides opportunities for school-agedyouth to participate in on-the-land skills training, including country food preparation. While providing an important opportunity for enhancing community access to country foods and fostering traditional land skills and knowledge acquisition, such programs create the context for effective transfer of traditional knowledge across generations (Wesche, O'Hare-Gordon, Robidoux, \& Mason, 2016).

A number of other school-based activities also contribute to youth engagement around food procurement and nutrition. Schools in the ISR offer opportunities for students to engage in on-the-land harvesting and engagement with Elders around country food butchering and preparation techniques. Additionally, aspects of local food production are integrated into parts of the educational curriculum (e.g. class greenhouse visits in Inuvik).

\section{Food security initiatives in the ISR: Cross-cutting themes}

This study underscores the fact that addressing food security through programming and other initiatives is multi-faceted and extremely complex. A number of important initiative-related themes emerge from this cross-scale synthesis, including: orientation with respect to food security pillars, scope and scale, demographic targeting, funding, monitoring and evaluation for evidence-based policy-making, and implications for food security strategies. These are discussed below.

\section{Addressing the four pillars of food security}

The pillars of food security - availability, access, quality and utilization - are not equally addressed by the programs identified in this review. The majority of programs aim to increase access to food, either by promoting financial access to market or country food, or by providing direct access to food through food distribution programs.

A much smaller number of programs address the fundamental pillar of food availability. Regarding market food, NNC's objective is to promote the availability of affordable healthy food in remote communities, but its current structure limits the extent to which program outcomes are evaluated (Galloway, 2017). For country food, this review did not identify any programs that 
specifically address the availability of relevant species; this limitation reinforces the importance of improving conceptual and practical linkages between wildlife management and health by applying a food security lens. Regarding locally-produced food, only a limited number of programs promote local agriculture or other local food production initiatives.

Most of the programs under theme 2 (Health and education programs) and theme 3 (Community wellness and intergenerational knowledge sharing) address food utilization in parallel to food access.

\section{Scope and scale of food security initiatives}

Food security initiatives identified in this review vary in both spatial and temporal scale. In the ISR, there exists a continuum of approaches, from short-term hunger-mitigation strategies (e.g. food banks, soup kitchens), to longer-term programs and strategies that target the systemic causes of food insecurity (e.g. through capacity building, community economic development, and infrastructure improvements).

While some programs address a specific determinant of food insecurity (for example, access to nutritious market foods via fiscal subsidies), many programs respond broadly to community needs, where the food security focus is implicit. For instance, many programs work holistically to encourage community wellness through youth engagement, intergenerational knowledge exchange, skills development, and sharing. Over the long term, such programs may also foster improved nutrition, health and food security by empowering communities to build resilient food systems.

Initiatives to support food security may be implemented at various levels of organization by any number of actors, including non-governmental, community, and stakeholder organizations/agencies. In the ISR, although federal initiatives (e.g. NNC, CPNP) have focused largely on increasing affordable access to nutritious market foods and health promotion, community-based priorities tend to emphasize access to country food, and this is reflected in a number of territorial, regional and locally-administered programs.

\section{Demographic targeting}

While some ISR programs serve all residents (e.g. the NNC subsidy) or a sub-set of interested residents (e.g. community greenhouses), many are delivered in targeted settings (e.g. Elder programs in community centers) and service a particular segment of the population (e.g. children, low income families). Generally, food security initiatives target vulnerable demographics including children and youth (e.g. school meal programs), pregnant women and infants (e.g. CPNP), single mothers, Elders, and households with no active hunter (e.g. community freezers).

Effective targeting is fundamental to enhancing food security among the most vulnerable subpopulations (Barrett, 2002) and is often explicitly considered in food program design and evaluation. Despite the widespread practice of program targeting, the literature suggests that in 
practice targeting may not always be feasible or desirable (Barrett, 2002), and it may also restrict access to individuals who would benefit from program inclusion. For instance, individuals who are middle aged or homeless are often overlooked in northern food support programs (Ford, Lardeau et al., 2013). Preferential support for certain groups may also happen implicitly, based on the locally-determined allocation of often scarce resources. Furthermore, it is important that any program targeting extends beyond identifying segments of the population and their needs to facilitating program access and awareness among such individuals. For instance, delivery or transportation services may benefit participants with limited mobility (The Food Security Network of Newfoundland \& Labrador, 2010).

\section{Funding}

Continuity in program leadership and funding represents an important challenge for northern food program design (The Food Security Network of Newfoundland \& Labrador, 2010). The majority of food security programs in the ISR operate based on government funding at various levels (e.g. national, territorial and regional). In the absence of formal government funding, community food programs generally depend on voluntary community support and donations. As such, the scarcity of available volunteers and the rate of volunteer satiation (whereby the same individuals volunteer for multiple programs) limits program effectiveness at the local scale (The Food Security Network of Newfoundland \& Labrador, 2010).

The provenance of program funding and administration may also affect program scope, targeting and governance. For example, the Arctic Char Distribution Project (in Nunavik, Quebec), which provided free fish to pregnant women in need, was perceived to have strong community-based value as it was locally-conceived, rather than a federal initiative (Gautier, Pirkle, Furgal, \& Lucas, 2016). The broader literature on food assistance programs has yielded only a vague understanding of the "appropriate blend" of private and public institutions and interventions (Barrett, 2002).

\section{Monitoring and evaluation}

During our review, we found limited evidence of monitoring and evaluation regarding identified initiatives. While NNC comprises an annual monitoring process, it is criticized by Northern residents as having limited capacity to assess effectiveness (Rennie, 2014) and by the Auditor General of Canada for its lack of transparency (Office of the Auditor General of Canada, 2014). While the Government of Canada has committed to improving NNC's monitoring and conducted a significant stakeholder engagement process in 2016, changes have yet to be undertaken.

Program monitoring and evaluation are required to inform policymakers and the public about the effectiveness of public investment and actions in solving social problems (van der Veen \& Gebrehiwot, 2011). It is important to document both operational and impact-related outcomes. In other words, monitoring processes that document program-level outputs (e.g. the 
volume of food delivered or the number of people serviced by the program) and program impact evaluations that assess the extent to which a program mediates changes in food security conditions (Riely, Mock, Cogill, Bailey, \& Kenefick, 1999) should be used complementarily.

The empirical documentation of the effect of food programs on food security (Bartfeld \& Ahn, 2011) has inherent challenges. These include: isolating the impact of a given program among individuals who engage in multiple programs; overcoming selection bias, since persons at greater risk of food insecurity are more likely to participate; and more fundamentally, determining effective indicators and metrics of food security (e.g. food expenditure, nutritional status, food security questionnaires). Local perspectives are key to this type of evaluation (Riches, 2003). Existing methodologies regarding participatory program planning and evaluation (Nichols, 2002; Whitmore, 1998) represent promising approaches for capturing the multidimensionality of Inuit food security.

\section{Designing integrated food security strategies}

Factors that influence food availability, access, quality and utilization in the Inuit food system do not exist in isolation, but rather interact over different spatial and temporal scales (Ford \& Beaumier, 2011). As such, a multidimensional continuum of initiatives is needed to address food insecurity, ranging from short-term mitigation activities to long-term organizational change and policy responses that focus on root causes (Council of Canadian Academies, 2014).

Our review shows that while a significant number of existing initiatives address aspects of food security in the ISR, they are often ad hoc and it is difficult to track the range of programs in operation at any one time. Furthermore, scalar mismatches appear to be common, where intentions at the program administration level do not address key local needs. At the regional scale, coordinated food security strategies that are developed through extensive community consultation and reflect local needs and priorities would help to guide decision-making and ensure that resources are used efficiently and effectively. Strategic planning around food security is currently underway at national, territorial and regional levels; as such, there is significant scope to align policy goals across scales at this critical juncture.

In Canada, the Inuit territory of Nunavut has followed such an approach, undertaking an extensive consultation process to develop a regional-scale food security strategy and action plan that recognizes the interdependent nature of market food, country food and locally-produced food in the food system (Nunavut Food Security Coalition, 2014). The resulting framework now helps to guide investments, resources and programming at both regional and local levels in Nunavut, and provides a useful model for other Inuit regions. Adopting a similar approach in the ISR would allow the identification and highlighting of Inuvialuit priorities to support the development of an integrated food security strategy. 


\section{Conclusion}

Food security is a complex and multi-faceted issue, and one that is particularly problematic in remote, northern communities. In the ISR, and elsewhere across the north, there are many initiatives underway that address the different food security pillars. While it is clearly important to understand program-level dynamics, a broader synthesis of initiatives at a regional scale offers key perspectives about how different food security challenges are being addressed and how initiatives interrelate.

Here we provide a framework for synthesizing information about a wide range of food security initiatives that can serve as a template for future data collection and longitudinal comparison in the ISR and elsewhere. This information can help in identifying gaps and opportunities for program development for under-addressed food security pillars and for underserved communities and segments of the population. It also supports decision-makers in aligning resources across sectors, and has implications for other northern regions and for currently developing policy frameworks at multiple scales.

Building on the current research, additional investigation into the costs of food security initiatives in relation to the spectrum of results achieved (from process outcomes, to health and population level effects) would be a useful next step. A comprehensive synthesis and evaluation of food-security related initiatives across Indigenous territories/regions across the north, to identify commonalities, efficiencies and gaps, would benefit the design (and redesign) of food security initiatives. Furthermore, work to identify mechanisms that strengthen the alignment of policy goals across scales could play a key role in supporting positive program outcomes.

This assessment highlights the importance of monitoring and evaluation to improve understandings of program effectiveness and complementarity. Furthermore, it highlights the important role of local perspectives and involvement in coordinated approaches for addressing food security. In the evolving landscape of Inuit food systems, broader-scale, holistic governance strategies can play a useful role in aligning local programming with priorities, policies and resources across scales. Such processes must be supported (or driven by) local and regional governance organizations.

Acknowledgements: We are grateful to our project participants for sharing their knowledge and time, and to ArcticNet and the Northern Scientific Training Program for project funding. We also thank two anonymous reviewers for their helpful feedback on an earlier draft.

\section{References}

Avard, E. (2015). Northern greenhouses: An alternative local food provisioning strategy for Nunavik (Doctoral dissertation). Québec, QC: Université Laval. Retrieved from http://theses.ulaval.ca/archimede/meta/31637 
Bains, A., Pakseresht, M., Roache, C., Beck, L., Sheehy, T., Gittelsohn, J., Corriveau, A., \& Sharma, S. (2014). Healthy Foods North improves diet among Inuit and Inuvialuit women of childbearing age in Arctic Canada. Journal of Human Nutrition and Dietetics, 27(s2), 175-185.

Barrett, C. B. (2002). Food security and food assistance programs. In B. L. Gardner \& G. C. Rausser (Eds.), Handbook of Agricultural Economics (Vol. 2, pp. 2103-2190). Amsterdam, the Netherlands: Elsevier.

Bartfeld, J. S., \& Ahn, H. M. (2011). The School Breakfast Program strengthens household food security among low-income households with elementary school children. Journal of Nutrition, 141(3), 470-475.

Beaumier, M. C., \& Ford, J. D. (2010). Food insecurity among Inuit women exacerbated by socio-economic stresses and climate change. Canadian Journal of Public Health, 101(3), 196-201.

Borré, K. (1991). Seal blood, Inuit blood, and diet: A biocultural model of physiology and cultural identity. Medical Anthropology Quarterly, 5(1), 48-62.

Boult, D. A. (2004). Hunger in the Arctic: Food (in)security in Inuit communities. Ottawa, ON: Ajunnginiq Centre, National Aboriginal Health Organization. Retrieved from https://foodsecurecanada.org/sites/foodsecurecanada.org/files/2004_inuit_food_security.pd f.

Burnett, K., Skinner, K., \& LeBlanc, J. (2015). From Food Mail to Nutrition North Canada: Reconsidering federal food subsidy programs for Northern Ontario. Canadian Food Studies, 2(1): 141-56.

Council of Canadian Academies. (2014). Aboriginal food security in northern Canada: An assessment of the state of knowledge. Ottawa, ON: CCA. Retrieved from http://www.scienceadvice.ca/en/assessments/completed/food-security.aspx.

Chan, L. (2012). Inuit Health Survey 2007-2008: Contaminant assessment in Nunavut. Nunavut Tunngavik Incorporated. Retrieved from http://www.tunngavik.com/blog/publications/inuit-health-survey-2007-2008-contaminantassessment-in-nunavut/

Chan, H. M., Kim, C., Khoday, K., Receveur, O., \& Kuhnlein, H. V. (1995). Assessment of dietary exposure to trace metals in Baffin Inuit food. Environmental Health Perspectives, 103(7-8), 740-746.

Chan, H. M. (1998). A database for environmental contaminants in traditional foods in northern and arctic Canada: Development and applications. Food Additives and Contaminants, 15(2), 127-134.

Chan, H. M. (2006). Food safety and food security in the Canadian Arctic. Meridian, Fall/Winter, $1-4$.

Chan, H. M., Fediuk, K., Hamilton, S., Rostas, L., Caughey, A., Kuhnlein, H. V., ... \& Loring, E. (2006). Food security in Nunavut, Canada: Barriers and recommendations. International Journal of Circumpolar Health, 65(5), 416-431. 
Collings, P. (2011). Economic strategies, community, and food networks in Ulukhaktok, Northwest Territories, Canada. Arctic, 64(2), 207-219

Collings, P., Marten, M. G., Pearce, T., \& Young, A. G. (2016). Country food sharing networks, household structure, and implications for understanding food insecurity in Arctic Canada. Ecology of Food and Nutrition, 55(1), 30-49.

Collings, P., Wenzel, G. W., \& Condon, R. G. (1998). Modern food sharing networks and community integration in the central Canadian Arctic. Arctic, 51(4), 301-314.

Condon, R. G., Collings, P., \& Wenzel, G. W. (1995). The best part of life: Subsistence hunting, ethnicity, and economic adaptation among young adult Inuit males. Arctic, 48(1), 31-46.

De Schutter, O. (2012). Report of the Special Rapporteur on the Right to Food: Addendum, Mission to Canada. Geneva, Switzerland: United Nations. Retrieved from http://www.srfood.org/images/stories/pdf/officialreports/20121224_canadafinal_en.pdf

Donaldson, S. G., Van Oostdam, J., Tikhonov, C., Feeley, M., Armstrong, B., Ayotte, P., ... \& Shearer, R. G. (2010). Environmental contaminants and human health in the Canadian Arctic. Science of the Total Environment, 408(22), 5165-5234.

Duhaime, G., \& Caron, A. (2012). Indices comparatifs des prix du Nunavik 2011. Quebec, QC: Université Laval. Retrieved from http://www.chaireconditionautochtone.fss.ulaval.ca/en/Publications.aspx

Duhaime, G., Chabot, M., \& Gaudreault, M. (2002). Food consumption patterns and socioeconomic factors among the Inuit of Nunavik. Ecology of Food and Nutrition, 41(2), 91-118.

Duhaime, G., Morin, A., Myers, H., Caulfield, R., Fréchette, P., \& St-Pierre, D. (2002). Food networks in the North American Arctic. In G. Duhaime (Ed.), Sustainable food security in the Arctic: State of knowledge (pp. 63-74). Edmonton, AB: Canadian Circumpolar Institute Press.

Egeland, G. M. (2010). Inuit Health Survey 2007-2008: Inuvialuit Settlement Region. Montreal, QC: Centre for Indigenous Peoples’ Nutrition and Environment.

Egeland, G. M. (2011). IPY Inuit Health Survey speaks to need to address inadequate housing, food insecurity and nutrition transition. International Journal of Circumpolar Health, 70(5), 444-446.

Egeland, G. M., Johnson-Down, L., Cao, Z. R., Sheikh, N., \& Weiler, H. A. (2011). Food insecurity and nutrition transition combine to affect nutrient intakes in Canadian Arctic communities. Journal of Nutrition, 141(9), 1746-1753.

Egeland, G. M., Williamson-Bathory, L., Johnson-Down, L., Sobol, L. (2011). Traditional food and monetary access to market-food: Correlates of food insecurity among Inuit preschoolers. International Journal of Circumpolar Health, 70(4), 373-383.

Enrg Research Group. (2016). Northern food retail data collection \& analysis. Ottawa, ON: Government of Canada. Retrieved from http://www.nutritionnorthcanada.gc.ca/eng/1424364469057/1424364505951 
Erber, E., Beck, L., Hopping, B. N., Sheehy, T., De Roose, E., \& Sharma, S. (2010). Food patterns and socioeconomic indicators of food consumption amongst Inuvialuit in the Canadian Arctic. Journal of Human Nutrition and Dietetics 23(s1), 59-66.

FAO. (1996). Rome declaration on world food security. Rome, Italy. Retrieved from http://www.fao.org/docrep/003/w3613e/w3613e00.htm

Fillion, M., Laird, B., Douglas, V., Van Pelt, L., Archie, D., \& Chan, L. H. (2014). Development of a strategic plan for food security and safety in the Inuvialuit Settlement Region, Canada. International Journal of Circumpolar Health, 73 (1), 25091.

Flowers, J., Nochasak, S., \& Jameson, K. (2010). NiKigijavut Hopedalimi “Our food in Hopedale”. Newfoundland \& Labrador: Food Security Network. Retrieved from http://www.foodsecuritynews.com/community-led-food-assessments.html.

Ford, J. D. (2009). Vulnerability of Inuit food systems to food insecurity as a consequence of climate change: A case study from Igloolik, Nunavut. Regional Environmental Change, 9(2), 83-100.

Ford, J. D., \& Beaumier, M. C. (2011). Feeding the family during times of stress: Experience and determinants of food insecurity in an Inuit community. The Geographical Journal, 177(1), 44-61.

Ford, J. D., Lardeau, M. P., Blackett, H., Chatwood, S., \& Kurszewski, D. (2013). Community food program use in Inuvik, Northwest Territories. BMC Public Health, 13(1), 970.

Ford, J. D., Lardeau, M.-P., \& Vanderbilt, W. (2012). The characteristics and experience of community food program users in Arctic Canada: A case study from Iqaluit, Nunavut. BMC Public Health, 12(1), 464.

Ford, J. D., Macdonald, J. P., Huet, C., Statham, S., \& MacRury, A. (2016). Food policy in the Canadian North: Is there a role for country food markets? Social Science \& Medicine, 152(2016), 35-40.

Ford, J. D., McDowell, G., Shirley, J., Pitre, M., Siewierski, R., Gough, W.A., ... \& Statham, S. (2013). The dynamic multiscale nature of climate change vulnerability: An Inuit harvesting example. Annals of the Association of American Geographers, 103(5), 1193-1211.

Ford, J. D., Smit, B., \& Wandel, J. (2006). Vulnerability to climate change in the Arctic: A case study from Arctic Bay, Canada. Global Environmental Change, 16(2), 145-160.

Furgal, C., \& Seguin, J. (2006). Climate change, health and vulnerability in Canadian northern Aboriginal communities. Environmental Health Perspectives, 114 (12), 1964-1970.

Galloway, T. (2014). Is the Nutrition North Canada retail subsidy program meeting the goal of making nutritious and perishable food more accessible and affordable in the North? Canadian Journal Public Health, 105(5), e395-e397.

Galloway, T. (2017). Canada's northern food subsidy Nutrition North Canada: A comprehensive program evaluation. International Journal of Circumpolar Health, 76(1), 1279451.

Gates, M., Hanning, R. M., Gates, A., McCarthy, D. D., \& Tsuji, L. J.S. (2013). Assessing the impact of pilot school snack programs on milk and alternatives intake in 2 remote First Nation communities in Northern Ontario, Canada. Journal of School Health, 83(2), 69-76. 
Gates, A., Hanning, R. M., Gates, M., Stephen, J., \& Tsuji, L. J. S. (2016). Four-year evaluation of a healthy school snack program in a remote First Nations community. Health Behavior and Policy Review, 3(3), 226-237.

Gautier, L., Pirkle, C. M., Furgal, C., \& Lucas, M. (2016). Assessment of the implementation fidelity of the Arctic Char distribution project in Nunavik, Quebec. BMJ Global Health, 1(3), e000093.

Giroux, M. A., Campbell, M., Dumond, M., \& Jenkins, D. (2012). Availability of caribou and muskoxen for local human consumption across Nunavut. Iqaluit, NU: Government of Nunavut. Retrieved from http://www.gov.nu.ca/sites/default/files/availability_of_caribou_and_muskoxen_for_local_ human_consumption_across_nunavut_2012.pdf

GNWT. (2013). Building on the strengths of northerners: A strategic framework toward the elimination of poverty in the NWT. Yellowknife, NT: Government of Northwest Territories. Retrieved from http://www.assembly.gov.nt.ca/sites/default/files/13-0606td_97-174.pdf

GNWT. (2014). Government of the Northwest Territories anti-poverty action plan. Building on the strengths of northerners 2014/15-2015/16. Yellowknife, NT: Government of Northwest Territories, Department of Health and Social Services. Retrieved from http://www.hss.gov.nt.ca/sites/www.hss.gov.nt.ca/files/resources/gnwt-anti-poverty-actionplan.pdf

GNWT. (2017). Northwest Territories agriculture strategy. The business of food: A Food Production Plan 2017-2022. Yellowknife, NT: Government of Northwest Territories, Department of Industry, Tourism and Investment. Retrieved from http://www.iti.gov.nt.ca/sites/www.iti.gov.nt.ca/files/agriculture_strategy.pdf

Gombay, N. (2009). Sharing or commoditising? A discussion of some of the socio-economic implications of Nunavik's Hunter Support Program. Polar Record, 45(233), 119-132.

Government of Canada. (2017). Eligible communities. Nutrition North Canada. Retrieved from http://www.nutritionnorthcanada.gc.ca/eng/1415540731169/1415540791407

Grey, S., \& Patel, R. (2015). Food sovereignty as decolonization: Some contributions from Indigenous movements to food system and development politics. Agriculture and Human Values, 32(3), 431-444.

Gunn, A., Russell, D. E., \& Eamer, J. (2011). Northern caribou population trends in Canada. Canadian biodiversity: Ecosystem status and trends 2010, Technical thematic report No. 10. Ottawa, ON: Canadian Councils of Resource Ministers. Retrieved from http://www.biodivcanada.ca/default.asp?lang=En\&n=F84ED404

Guyot, M., Dickson, C., Paci, C., Furgal, C., \& Chan, H.M. (2012). Local observations of climate change and impacts on traditional food security in two northern Aboriginal communities. International Journal of Circumpolar Health, 65(5), 403-415.

Health Canada. (2012). Household food insecurity in Canada in 2007-2008: Key statistics and graphics. Retrieved from http://www.hc-sc.gc.ca/fnan/surveill/nutrition/commun/insecurit/key-stats-cles-2007-2008-eng.php 
Hopping, B. N., Mead, E., Erber, E., Sheehy, C., Roache, C., \& Sharma, S. (2010). Dietary adequacy of Inuit in the Canadian Arctic. Journal of Human Nutrition and Dietetics, 23(sl), 27-34.

Huet, C., Rosol, R., \& Egeland, G. M. (2012). The prevalence of food insecurity is high and the diet quality poor in Inuit communities. Journal of Nutrition, 142(3), 541-547.

Inuit Tapiriit Kanatami (2014). Social determinants of Inuit health in Canada. Retrieved from https://www.itk.ca/wp-content/uploads/2016/07/ITK_Social_Determinants_Report.pdf

Inuvialuit Regional Corporation. (2013a). Aklavik community wellness plan. Inuvik, NWT. Retrieved from http://www.hss.gov.nt.ca/sites/hss/files/aklavik-community-wellness-planirc.pdf

Inuvialuit Regional Corporation. (2013b). Inuvik community wellness plan. Inuvik, NWT. Retrieved from http://www.hss.gov.nt.ca/sites/hss/files/irc-inuvik-community-wellnessplan.pdf

Inuvialuit Regional Corporation. (2013c). Paulatuk community wellness plan. Inuvik, NWT. Retrieved from http://www.hss.gov.nt.ca/sites/hss/files/paulatuk-community-wellnessplan.pdf

Inuvialuit Regional Corporation. (2013d). Sachs Harbour community wellness plan. Inuvik, NWT. Retrieved from http://www.hss.gov.nt.ca/sites/hss/files/sachs-harbourcommunitywellness-plan.pdf

Inuvialuit Regional Corporation. (2013e). Tuktoyaktuk community wellness plan. Inuvik, NWT. Retrieved from http://www.hss.gov.nt.ca/sites/hss/files/resources/tuktoyaktuk-communitywellness-plan.pdf

Inuvialuit Regional Corporation. (2013f). Ulukahaktok community wellness plan. Inuvik, NWT. Retrieved from http://www.hss.gov.nt.ca/sites/hss/files/resources/ulukhaktok-communitywellness-plan.pdf

Inuvialuit Regional Corporation. (2017). Inuvialuit lands. Retrieved from http://www.irc.inuvialuit.com/inuvialuit-lands

Islam, D., \& Berkes, F. (2016). Indigenous Peoples’ fisheries and food security: A case from northern Canada. Food Security, 8(4), 815-826.

Johnston, C., \& Williams, T. (2017). The Yellowknife Food Charter: Driving collaborative action for food security. Northern Public Affairs, (April), 27-32.

Kenny, T.-A., \& Chan, L. H. (2017). Estimating wildlife harvest based on reported consumption by Inuit in the Canadian Arctic. Arctic, 70(1), 1-12.

Kishigami, N. (2004). A new typology of food-sharing practices among hunter-gatherers, with a special focus on Inuit examples. Journal of Anthropological Research, 60(3), 341-358.

Kolahdooz, F., Butler, L., Lupu, M., Sheehy, T., Corriveau, A., \& Sharma, S. (2014). Assessment of dietary intake among Inuvialuit in Arctic Canada using a locally developed quantitative food frequency questionnaire. Journal of the American College of Nutrition, 33(2), 147-154. 
Kolahdooz, F., Pakseresht, M., Mead, E., Beck, L., Corriveau, A., \& Sharma, S. (2014). Impact of the Healthy Foods North nutrition intervention program on Inuit and Inuvialuit food consumption and preparation methods in Canadian Arctic communities. Nutrition Journal, 13(1), 68.

Kuhnlein, H. V., \& Chan, L. H. (2000). Environment and contaminants in traditional food systems of northern Indigenous Peoples. Annual Review of Nutrition, 20, 595-626.

Lambden, J., Receveur, O., \& Kuhnlein, H. V. (2007). Traditional food attributes must be included in studies of food security in the Canadian Arctic. International Journal of Circumpolar Health, 66(4), 308-319.

Lambden, J., Receveur, O., Marshall, J., \& Kuhnlein, H. V. (2006). Traditional and market food access in Arctic Canada is affected by economic factors. International Journal of Circumpolar Health, 65(4), 331-340.

Lardeau, M.P., Healey, G., \& Ford, J. D. (2011). The use of photovoice to document and characterize the food security of users of community food programs in Iqaluit, Nunavut. Rural and Remote Health, 11(2), 1680.

Levac, D., Colquhoun, H., \& O'Brien, K. K. (2010). Scoping studies: Advancing the methodology. Implementation Science, 5(1), 69.

Mackey, M. G. A., \& Orr, R. D. (1987). An evaluation of household country food use in Makkovik, Labrador, July 1980-June 1981. Arctic, 40(1), 60-65.

McTavish, K., Furgal, C., Popp, S., \& McCarney, P. (2012). Community-led food assessment for Inuit communities: Learning guide. Newfoundland and Labrador: Food Security Network. Retrieved from http://www.foodfirstnl.ca/our-resources/community-led-food-assessment

Mead, E., Gittelsohn, J., De Roose, E., \& Sharma, S. (2010a). Important psychosocial factors to target in nutrition interventions to improve diet in Inuvialuit communities in the Canadian Arctic. Journal of Human Nutrition and Dietetics, 23(S1), 92-99.

Mead, E., Gittelsohn, J., Kratzmann, M., Roache, C., \& Sharma, S. (2010b). Impact of the changing food environment on dietary practices of an Inuit population in Arctic Canada. Journal of Human Nutrition and Dietetics, 23(S1), 18-26.

Mead, E., Gittelsohn, J., Roache, C., Corriveau, A., \& Sharma, S. (2013). A community-based, environmental chronic disease prevention intervention to improve healthy eating psychosocial factors and behaviors in Indigenous populations in the Canadian Arctic. Health Education \& Behavior, 40(5), 592-602.

Meakin, S., \& Kurvits, T. (2009). Assessing the impacts of climate change on food security in the Canadian Arctic. Norway: GRID-Arendal. Retrieved from http://stage.cakex.org/sites/default/files/GRID-Arendal_food.security.pdf

Morrison, D. (2011). Indigenous food sovereignty: A model for social learning. In Wittman, H., Desmarais, A. A., \& Wiebe, N. (Eds.), Food sovereignty in Canada: Creating just and sustainable food systems (pp. 97-113). Winnipeg, MN: Fernwood Publishing. 
Myers, H. (2002). The changing food economy in Nunavut: Will country food stores secure Nunavut's food supply? In: Duhaime, G. (Ed.), Sustainable Food Security in the Arctic: State of Knowledge (pp. 95-101). Edmonton, AB: Canadian Circumpolar Institute Press.

Myers, H., Powell, S., \& Duhaime, G. (2004). Setting the table for food security: policy impacts in Nunavut. Canadian Journal of Native Studies, 24(2), 425-445.

Nancarrow, T. L., \& Chan, L. H. (2010). Observations of environmental changes and potential dietary impacts in two communities in Nunavut, Canada. Rural and Remote Health, 10(2), 1370.

Natcher, D. C. (2009). Subsistence and the social economy of Canada's Aboriginal North. The Northern Review, 30(Spring), 83-98.

Nichols, L. (2002). Participatory program planning: Including program participants and evaluators. Evaluation and Program Planning, 25(1), 1-14.

Nunavut Food Security Coalition. (2014). Nunavut food security strategy and action plan 201416. Retrieved from http://foodsecurecanada.org/sites/foodsecurecanada.org/files/nunavutfoodsecuritystrategy_ english_0.pdf

NWT Bureau of Statistics. (2016). Summary of NWT community statistics - 2016. Bureau of Statistics, Government of the Northwest Territories. Retrieved from http://www.statsnwt.ca/community-data/index.html

Office of the Auditor General of Canada. (2014). Chapter 6 - Nutrition North Canada Aboriginal Affairs and Northern Development Canada. Retrieved from http://www.oagbvg.gc.ca/internet/English/parl_oag_201411_06_e_39964.html

Olson, C. M. (1999). Nutrition and health outcomes associated with food insecurity and hunger. Journal of Nutrition, 129(2S), 521-524.

Organ, J. (2012). Community freezers supporting food security: Perspectives from residents of Nain, Nunatsiavut (Master's thesis). Dalhousie University, Halifax, NS. Retrieved from http://www.bac-lac.gc.ca/eng/services/theses/Pages/theses-canada.aspx

Organ, J., Castleden, H., Furgal, C., Sheldon, T., \& Hart, C. (2014). Contemporary programs in support of traditional ways: Inuit perspectives on community freezers as a mechanism to alleviate pressures of wild food access in Nain, Nunatsiavut. Health \& Place, 30, 251-259.

Parker, C. (2016). Examining the vulnerability of an Inuit food system to climate change in the context of climatic and non-climatic stressors: A case study of Ulukhaktok, NT (Master's thesis). Guelph, ON: University of Guelph. Retrieved from https://atrium2.lib.uoguelph.ca/xmlui/handle/10214/10028

Pearce, T., Wright, H., Notaina, R., Kudlak, A., Smit, B., Ford, J. D., \& Furgal, C. (2011). Transmission of environmental knowledge and land skills among Inuit men in Ulukhaktok, Northwest Territories, Canada. Human Ecology, 39(3), 271-288.

Power, E. M. (2008). Conceptualizing food security for Aboriginal people in Canada. Canadian Journal of Public Health, 99(2), 95-97. 
Rennie, S. (2014, December 21). Nutrition North food subsidy program: What went wrong. CBC News. Retrieved from http://www.cbc.ca/news/canada/north/nutrition-north-food-subsidyprogram-what-went-wrong-1.2880756

Riches, G. (2003). Food banks and food security: Welfare reform, human rights and social policy. Lessons from Canada? Social Policy \& Administration, 36(6), 648-663.

Riely, F., Mock, N., Cogill, B., Bailey, L., \& Kenefick, E. (1999). Food security indicators and framework for use in the monitoring and evaluation of food aid programs. Washington, DC: Food and Nutrition Technical Assistance Project (FANTA). Retrieved from https://reliefweb.int/sites/reliefweb.int/files/resources/0162465863FC5DD0C1256DEC004 94A30-USAID_foodIndicator_1999.pdf

Rosol, R., Huet, C., Wood, M., Lennie, C., Osborne, G., \& Egeland, G. M. (2011). Prevalence of affirmative responses to questions of food insecurity: International Polar Year Inuit Health Survey, 2007-2008. International Journal of Circumpolar Health, 70(5), 488-497.

Rosol, R., Powell-Hellyer, S., \& Chan, L. H. (2017). Impacts of decline harvest of country food on nutrient intake among Inuit in Arctic Canada: Impact of climate change and possible adaptation plan. International Journal of Circumpolar Health, 75(1), 31127.

Saudny, H., Egeland, G. M., \& Leggee, D. (2012). Design and methods of the Adult Inuit Health Survey 2007-2008. International Journal of Circumpolar Health, 71(1), 19752.

Searles, E. (2002). Food and the making of modern Inuit identities. Food and Foodways, 10(1-2), 55-78.

Searles, E. N. (2016). To sell or not to sell: Country food markets and Inuit identity in Nunavut. Food and Foodways, 24(3-4), 194-212.

Seligman, H. K., Laraia, B. A., \& Kushel, M. B. (2010). Food insecurity is associated with chronic disease among low-income NHANES participants. Journal of Nutrition, 140(2), 304-310.

Sharma, S. (2010). Assessing diet and lifestyle in the Canadian Arctic Inuit and Inuvialuit to inform a nutrition and physical activity intervention programme. Journal of Human Nutrition and Dietetics, 23(S1), 5-17.

Sharma, S., Gittelsohn, J., Rosol, R., \& Beck, L. (2010). Addressing the public health burden caused by the nutrition transition through the Healthy Foods North nutrition and lifestyle intervention programme. Journal of Human Nutrition and Dietetics, 23(S1), 120-127.

Skinner, K., Burnett, K., Williams, P. L., Martin, D., Stothart, C., LeBlanc, J., .... \& Sheedy, A. (2016). Challenges in assessing food environments in northern and remote communities in Canada. Canadian Journal of Public Health, 107 (S1), eS60-eS63 .

Skinner, K., Hanning, R. M., \& Tsuji, L. J. (2014). Prevalence and severity of household food insecurity of First Nations people living in an on-reserve, sub-Arctic community within the Mushkegowuk Territory. Public Health Nutrition, 17(1), 31-39.

Skinner, K., Hanning, R. M., Metatawabin, J., Martin, I. D, Tsuji, L. J. S. (2012). Impact of a school snack program on the dietary intake of grade six to ten First Nation students living in a remote community in northern Ontario, Canada. Rural and Remote Health. 12: 2122. 
Stuff, J. E., Casey, P. H., Szeto, K. L., Gossett, J. M., Robbins, J. M., Simpson, P. M., ... \& Bogle, M. L. (2004). Household food insecurity is associated with adult health status. Journal of Nutrition, 134(9), 2330-2335.

Theriault, S. (2011). The food security of the Inuit in times of change: Alleviating the tension between conserving biodiversity and access to food. Journal of Human Rights and the Environment, 2(2), 136-156.

Todd, Z. S. C. (2010). Food security in Paulatuk, NT-opportunities and challenges of a changing community economy (Master's thesis). Edmonton, AB: University of Alberta. Retrieved from https://era.library.ualberta.ca/files/dr26xx46t\#.Wmem3XIG2Uk

Usher, P. J. (1976). Evaluating country food in the northern native economy. Arctic, 29(2): 105120.

van der Veen, A., \& Gebrehiwot, T. (2011). Effect of policy interventions on food security in Tigray, Northern Ethiopia. Ecology and Society, 16(1) 18.

Van Oostdam, J., Donaldson, S. G., Feeley, M., Arnold, D., Ayotte, P., Bondy, G., ... \& Kalhok, S. (2005). Human health implications of environmental contaminants in Arctic Canada: A review. Science of the Total Environment, 351(1), 165-246.

Weiler, A. M., Hergesheimer, C., Brisbois, B., Wittman, H., Yassi, A., \& Spiegel, J. M. (2015). Food sovereignty, food security and health equity: A meta-narrative mapping exercise. Health Policy and Planning, 30(8), 1078-1092.

Wenzel, G. W. (1991). Animal rights, human rights: Ecology, economy, and ideology in the Canadian Arctic. Toronto, ON: University of Toronto Press.

Wenzel, G. W. (1995). Ningiqtuq: Resource sharing and generalized reciprocity in Clyde River, Nunavut. Arctic Anthropology, 32(2), 43-60.

Wesche, S. D., \& Chan, H.M. (2010). Adapting to the impacts of climate change on food security among Inuit in the Western Canadian Arctic. EcoHealth, 7(3), 361-373.

Wesche, S. D., O'Hare-Gordon, M. A. F., Robidoux, M. A., \& Mason, C. W. (2016). Land-based programs in the Northwest Territories: Building Indigenous food security and well-being from the ground up. Canadian Food Studies, 3(2), 23-26.

Whitmore, E. (1998). Understanding and practicing participatory evaluation: New Directions for Evaluation (vol. 80). San Francisco, CA: Jossey-Bass.

Whittles, M. (2014). Economic development as if culture matters: Inuvialuit wild game harvesting community-based economic development and cultural maintenance in the Western Arctic. Journal of Aboriginal Economic Development, 4(2), 129-140.

Willows, N. D. (2005). Determinants of healthy eating in Aboriginal Peoples in Canada: The current state of knowledge and research gaps. Canadian Journal of Public Health, 96(s3), S32-S36.

Willows, N. D., Veugelers, P., Raine, K., \& Kuhle, S. (2011). Associations between household food insecurity and health outcomes in the Aboriginal population (excluding reserves). Health Reports, 22(2), 15-20. Retrieved from https://www.statcan.gc.ca/pub/82-003x/2011002/article/11435-eng.htm 
Willows, N. D. (2005). Determinants of healthy eating in Aboriginal Peoples in Canada: The current state of knowledge and research gaps. Canadian Journal of Public Health, 96(s3), S32-S36.

Zotor, F., Sheehy, T., Lupu, M., Kolahdooz, F., Corriveau, A., \& Sharma, S. (2012). Frequency of consumption of foods and beverages by Inuvialuit adults in Northwest Territories, Arctic Canada. International Journal of Food Sciences and Nutrition, 63(7), 782-789. 